\title{
DEVELOPMENT OF A SPIN-INJECTION DEVICE UTILISING GADOLINIUM NITRIDE
}

\author{
by \\ KIRA MICHELLE PITMAN
}

\begin{abstract}
A thesis
submitted to the Victoria University of Wellington in fulfilment of the requirements for the degree of Master of Science
\end{abstract}

Victoria University of Wellington

2020 



\section{Abstract}

In this thesis, the first steps in creating a realisable spin-injection transistor using ferromagnetic semiconductor electrodes are detailed. A spin-injection device utilising the ferromagnetic semiconductor gadolinium nitride has been designed, fabricated and electrically tested. In addition, an experimental setup for future measurements of a spin current in spin-injection devices was adapted to our laboratory based off one developed by the Shiraishi group at Kyoto University. Issues encountered during fabrication were identified, and an optimal method for fabricating these devices was determined. Gadolinium nitride and copper were used to make the devices on $\mathrm{Si} / \mathrm{SiO}_{2}$ substrates.

The electrical integrity and applicability of the devices for future measurements of injected spin-current was determined through electrical device testing. Resistance measurements of electrical pathways within the device were undertaken to determine the successful deposition of the gadolinium nitride and copper. IV measurements to determine if the devices could withstand the current required for spin current measurements were done. The durability of the devices through multiple measurement types was observed. It was determined that although spin-injection devices utilising gadolinium nitride can be successfully fabricated, more work needs to be done to ensure that the electrical pathways through the copper and gadolinium nitride can be consistently reproducable to allow spin-injection measurements to be done. 


\section{Acknowledgements}

Thank you to everyone who has contributed directly or indirectly to me finishing my MSc.

Firstly, thank you to the entire Spintronics research group and the Cleanroom group. I have valued every science and not-science discussion, and they have been a central part of my MSc experience. Thank you to Denis Lefebvre for growing GdN on all but one of my wafers, and thank you to Franck Natali for growing the final one. Thank you to everyone in the Robinson Research Institute based at Gracefield that assisted me in my research. Thank you to Guy Dubuis for your help in making my breakout box and Bob Buckley for letting me use your office and your encouragement in my project.

Thank you to the Shiraishi group at Kyoto University for not only welcoming me into your research group and labs for two weeks but also for your assistance and contribution to the LabVIEW program and setting up the measurement system.

Thank you to Petrik Galvosas and the entire NMR group for always welcoming me into your lab.

Thank you so much to my supervisors Simon Granville and Ben Ruck. I am so thankful for having been able to do my Masters, and I am beyond grateful for the patience and support you have both given me in my project, particularly in allowing me the space I needed to prioritise myself ahead of my degree.

Thank you to Felicia Ullstad for your emotional support, assistance with latex, and supplying me with zucchini. Thank you to Sam Devese for your seemingly endless patience with every single problem I encountered with my photolithography and for 
being an absolute all-round star. Erica Cassie, thank you for your help with teaching me most things in the cleanroom and your encouragement to prioritise myself over my work. I also deeply appreciate your contribution to making sure the office had more than one Gay, all of our office outings and for introducing me to refried beans.

Sarah Stevenson, thank you for your emotional support through not only my Masters but the last three years of my tertiary education experience. You have been an integral part of my post-graduate education, and I cannot thank you enough for your guidance and support.

Thank you to Alisdair Armstrong for introducing me to rock climbing, and for having a genuine enthusiasm for my running, research, and overall wellbeing (we love that arts/science solidarity). And finally, thank you to Tori Fernandes for your impeccable emotional support over the last 8 months, you and your burritos have been absolute rock-solid contributors to me not dropping out.

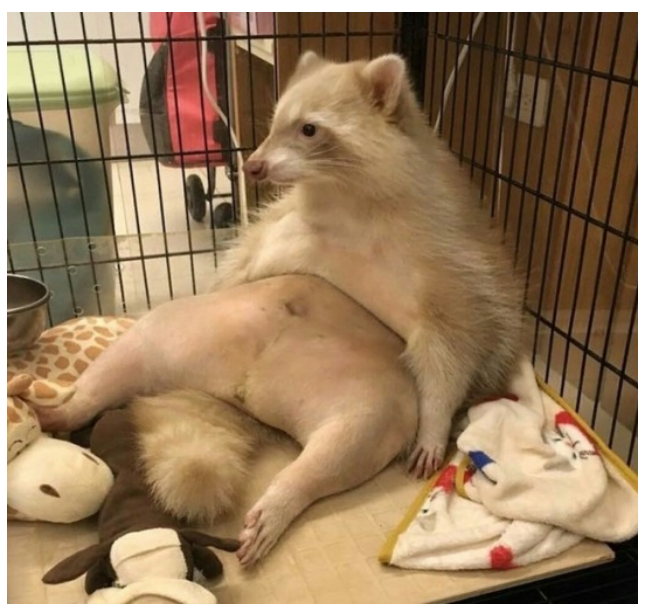

(a) raccoon 1

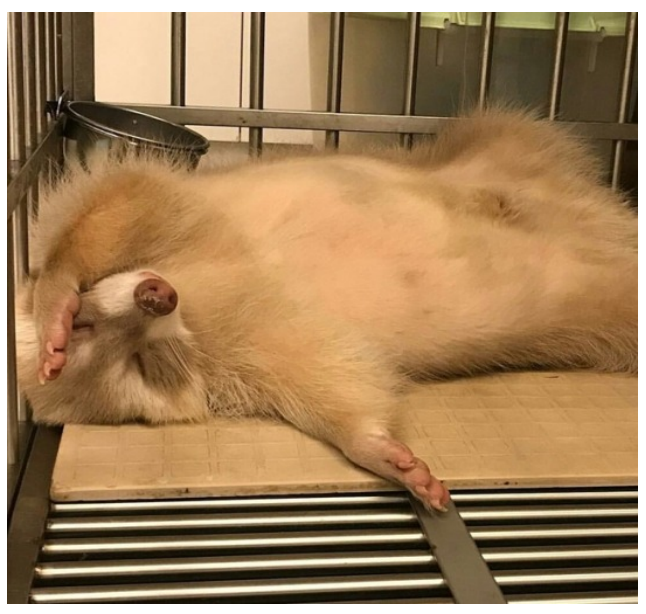

(b) raccoon 2

Figure 1: The thesis raccoons of detesting your writing slightly less. 


\section{Contents}

1 Introduction 1

2 Theory 5

2.1 Introduction to spintronics . . . . . . . . . . . . 5

2.2 Conventional Electronic Transistor . . . . . . . . . . . . . . . 7

2.3 Spin transistor . . . . . . . . . . . . . . . 8

2.4 The conductivity mismatch problem . . . . . . . . . . . . 11

2.4.1 Solving the conductivity mismatch problem . . . . . . . . . . . 12

2.5 Rare-earth Nitrides . . . . . . . . . . . . . . . . . . . . . 13

2.6 Spin-injection measurement . . . . . . . . . . . . . . 15

3 Experimental Methods $\quad 21$

3.1 Sample fabrication . . . . . . . . . . . . . . . . 21

3.1 .1 Photolithography . . . . . . . . . . . . . . 22

3.1.2 Contact pad deposition . . . . . . . . . . . . . . 24

3.1.3 Patterning of GdN electrodes . . . . . . . . . . . . . . 26

3.2 Characterisation Equipment . . . . . . . . . . . . . . . 28

3.3 Spin-injection measurement system . . . . . . . . . . . . . . 30

4 Results and Discussion $\quad 35$

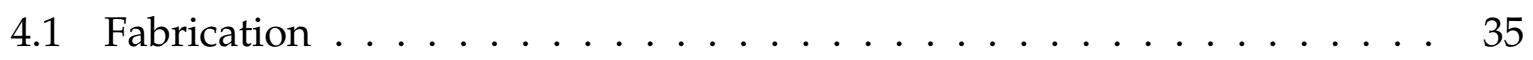

4.1 .1 GdN growth results . . . . . . . . . . . . . . . 35

4.1.2 Fabrication results for $10 \mu m, 5 \mu m$, and $2 \mu m$ channel lengths . . . 37

4.1.3 XRD characterisation for GdN electrodes . . . . . . . . . . . 38

4.1.4 Fabrication results discussion . . . . . . . . . . . . . 39

4.1.5 GdN and copper contact results discussion . . . . . . . . . . . 39

4.2 Device testing . . . . . . . . . . . . . . . . 40

4.2.1 Resistance measurements . . . . . . . . . . . . . 41 
4.2 .2 IV measurements . . . . . . . . . . . . . . . . . . . . . 44

4.2.3 Discussion of electrical testing results . . . . . . . . . . . . . . 44

4.2 .4 Durability of samples in testing . . . . . . . . . . . . . . 46

5 Conclusions and Future Work 49 


\section{Chapter 1}

\section{Introduction}

Climate change is the greatest problem facing the world today. As information becomes more globally attainable, huge amounts of energy are required to process the inundation of data. But the facilities where this processing is done consume a lot of energy. Data centres account for 3\% [1] of global electricity - approximately 416.2 terawatt-hours, more than the total energy consumption of the UK. They also account for $2 \%$ of greenhouse gas emissions [2] [3], equivalent to the carbon footprint of the entire airline industry. The energy requirements of data centres are doubling every 4 years and are expected to triple within the next decade [1].

Lowering the energy consumption of data centres will decrease the environmental impact and put less strain on worldwide energy production. One current possibility is to limit internet use and access. However, there are huge benefits to healthcare, education, and the economy with accessibility to information so a favourable alternative is to develop technology that will not only cope with the continual increase in data but also be energetically sustainable.

Developing devices where memory storage and retrieval capabilities are more energy efficient is necessary to curb the growing energy consumption of data centres. A prominent component of the technology used to process information is the transistor. Transistors are arguably one of the most important inventions of the 20th century. They are essential to integrated circuits, amplification, and memory - a 1GB memory device contains at least one billion transistors. The majority of our technology relies on transistors, so decreasing the energy consumption required to use them is necessary for 
improving the environmental sustainability of data centres, and the IT industry as a whole. The advancement of transistors based on electronic charge is slowing and will plateau over the next decade [4]. Therefore there is a need for a new type of transistor; one that has sustainable energy requirements and the potential to exceed the technological capabilities of current electronic devices.

A new type of transistor with potentially lower energy consumption is the spin transistor. A spin transistor is one that relies on the flow of spins of the electron to switch between states instead of the charge current. This flow of spins is referred to as the spin current. Spin injection transistors whose spin current travels between ferromagnetic source and drain electrodes by a non-magnetic conducting channel were first proposed by Datta and Das in 1990 [5]. Since then, there have been further developments in both the theoretical design of spin injection transistors [6] [7] and experimental work to build them, such as one using Cobalt as the ferromagnetic electrodes which allow for detection and controlled precession of spins in semiconductors which are also compatible with existing manufacturing processes using silicon and germanium [8].

As spin injection transistors rely on the orientation of the electron spin in the device, rather than the movement of electronic charges, they are part of the family of spintronic devices. Spintronics is a branch of technology which relies on the manipulation of the spins of particles, as opposed to the electrical charge [9]. This reliance on the spin of the electrons in the spin injection transistor means that the states of the system can be maintained without constant energy input. Consequently, spin injection transistors have the potential to be less energy demanding than their conventional electronic counterparts.

For a spin injection transistor to be realised, the spins need to be injected from the ferromagnetic electrodes into the semiconducting non-magnetic conducting channel at a high enough efficiency to provide a measurable spin polarised current. If the two materials used are similar in conductivity, they have high spin injection efficiency [10]. As most ferromagnetic materials are metallic, there is often a substantial conductivity mismatch between the ferromagnetic electrodes and the semiconductor used for the non-magnetic conducting channel. However, if materials are used that have similar 
conductivities, this conductivity mismatch problem could be solved.

The making of a spin-injection transistor is a very ambitious goal and one that will be realised over several years. In this thesis, I have developed a spin injection structure using a rare-earth nitride as the electrode material, and a copper as the non-magnetic material for the conducting channel and contact pads. Some RENs are ferromagnetic semiconductors and have tunable physical properties allowing them to seamlessly match with other materials in the device [11]. RENs consist of a Lanthanide 3- ion, and a Nitrogen 3+ ion, have tunable conductivity, and become ferromagnetic at low temperatures, allowing for potential high spin injection when acting as electrodes. RENs also have the advantage of being compatible with existing semiconductor technology bases. In producing a spin-injection device based on RENs, I have used Gadolinium Nitride $(\mathrm{GdN})$ for the ferromagnetic electrodes and copper for the transport channel.

Patterning with RENs is challenging, and using them in a spin injection transistor has not been previously explored. This research was focused on the first step of many - developing a proof of concept spin device using RENs as the ferromagnetic material. Future iterations of the REN based spin-injection device can use a semiconductor as the conducting channel material. With both the spin injector and channel materials made of semiconductors, it will be possible to match the conductivity of the materials of the transport channel, eliminating the conductivity mismatch problem and allowing a highly efficient injection of spin.

This thesis is split into six sections, with this introduction being the initial. Second is a theory section where the physics behind spintronics, spin transistors, and RENs are explored, along with a mathematical look at the origin of the measurements taking place. Third is the experimental methods section where an overview of the device growth processes and the measurement setup and techniques are discussed. Next the experimental results are shown, followed by a discussion in the penultimate section. Finally, the conclusions of this project are summarised and further research pathways are explored. 


\section{Chapter 2}

\section{Theory}

In this section I will provide an introduction to spintronics, explain the conventional electronic transistor, and how the spin transistor builds on it. The challenges being faced acheiving a working and commercially viable spin transistor will be discussed, as well as an overview of my chosen REN, GdN. Finally, an outline of the measurement techniques used to determine the presence or absence of a spin current is detailed.

\subsection{Introduction to spintronics}

Spintronics is a field of technology that is concerned with manipulating the spin of the electron. Spintronic devices can rely purely on spin or can be used together with the electron charge to transmit and manipulate information. There are many potential applications of such technology, such as quantum computers, spin-logic devices, and the spin transistor [9] [12][13].

The first spintronics technology was magnetic computer hard drives developed through the use of giant magnetoresistance (GMR). Discovered in 1988 [14][15], GMR is a change in resistance of $10-20 \%$ for a thin film of alternating ferromagnetic and non-magnetic layers in a magnetic field. This originates from the different scattering rates that electrons passing through the ferromagnetic layers have. These changes in resistance are large enough to form easily measurable electrical resistance states of the material; one that has high resistance, and one that has low. The scattering rate and electrical resistance of the two states are dependent on the relative orientation of the 
spin to the magnetisation of the ferromagnetic and non-magnetic layers. The high resistance state corresponds to an antiparallel alignment of the magnetisation of the ferromagnets and the low resistance state to parallel alignment of the ferromagnets. Therefore, by using a magnetic field to change the magnetic orientation of one layer, the state of the system can be changed. Hence, technology that relies on the spin of the electron can be developed.

Within a computer, there are three main essential types of device: long term storage, short term memory, and logic. Currently, devices rely on electronically based random access memory (RAM). RAM is where the data that is actively being used by the computer is stored. The capacitors used for RAM leak their charge and require a nearly constant power supply to maintain their charged state [16]. One application of spintronics is looking to create energy-efficient magnetoresistance random access memory (MRAM) for short term memory [17].

Information storage and retrieval in magnetic devices is performed by applying a magnetic field to reorientate the magnetic domains, not to maintain them. Just as with GMR, the state of the magnetic device is dependent on the relative orientation of the layers within the device. Once this orientation has been set, it stays in that state without needing to be actively powered. This makes MRAM a non-volatile type of memory. Hence, MRAM could eliminate this excess power consumption of electronic RAM, as applied power is only required for switching between or reading states, not maintaining them.

Spintronics also has potential applications in logic, in particular in the transistors that make up the core processors [18][17]. However, this has yet to be realised for industrial use. To date, logic components have been based on semiconductor technology, which is reaching its limits [4]. Consequently, alternative technologies for logic, including spin-based logic such as spin transistors are of great interest.

A spin injection transistor only requires energy to change or read states, not to maintain them and can be capable of maintaining that state even when the device is turned off. Without this need for constant input energy, the overall static power use could be lower than that of a conventional electronic transistor. 


\subsection{Conventional Electronic Transistor}

Electronic transistors are divided into two groups: bipolar junction transistors, and field-effect transistors. Bipolar junction transistors (BJTs) consist of two junction diodes formed by having either a p-type semiconductor between two n-type semiconductors or the other way around. The very thin base layer is sandwiched between the collector and emitter (see figure 2.1a). BJTs are current-controlled and are commonly used as amplifiers or switches. There are two currents within a BJT; a small one that goes between the emitter and base, and a larger one that goes between the emitter and collector. This small current controls the larger current by varying how many electrons travel to/from the emitter, and hence can turn the BJT on and off [19].

Field-effect transistors (FETs) have a source, gate, and drain (see figure 2.1b). In figure $2.1 \mathrm{~b}$ we can also see that there are three regions in the body of the FET: one $p$, and two $\mathrm{n}$. The $\mathrm{p}$ and $\mathrm{n}$ regions correspond to a $\mathrm{p}$-type and $\mathrm{n}$-type doped semiconductor. This semiconductor is commonly silicon, with p-type normally being doped by $\mathrm{B}, \mathrm{Ga}$, In, or $\mathrm{Al}$, and n-type using $\mathrm{P}, \mathrm{As}, \mathrm{Sb}, \mathrm{Bi}$, or $\mathrm{Li}[20]$.

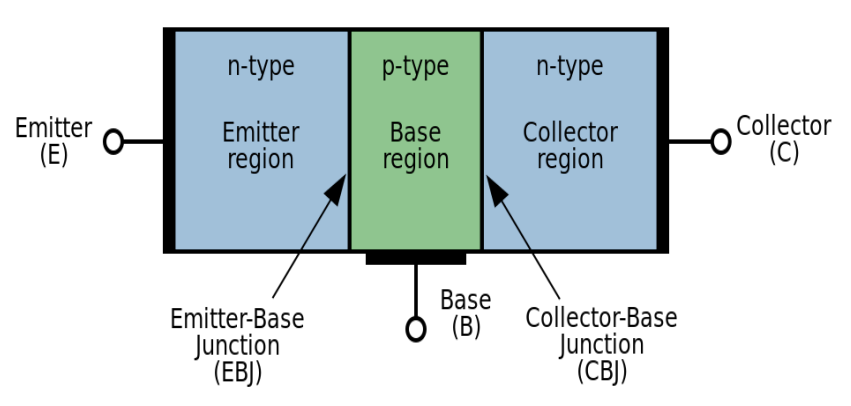

(a) Bipolar junction transistor sourced from [21]

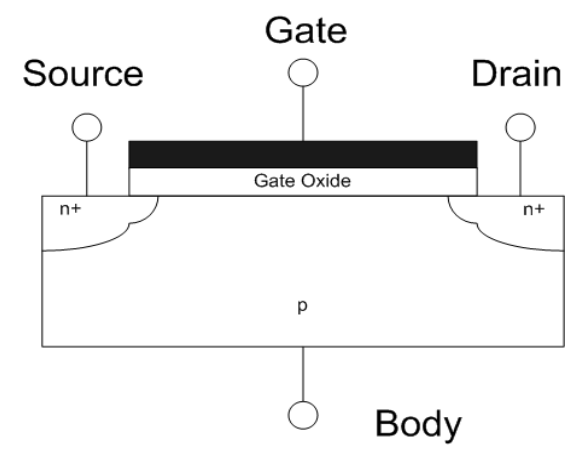

(b) Field effect transistor sourced from [22]

Figure 2.1: BJT and FET

In a FET, charge carriers flow from the source electrode to the drain electrode. The gate is an electrode through which a voltage can be applied that controls the flow of charge carriers between the source and drain electrodes (see figure 2.1b). Two states 
that are dependent on the gate voltage applied can be achieved: one where charge flows, and one where it doesn't. Many different FETs have been developed since the initial junction FET, including the MOSFET [23].

Switching the transistor between allowing charge carriers to flow from the source to drain, and preventing them from flowing, is controlled by the gate electrode. The path between the source and drain electrodes is known as the conducting channel. By applying a voltage through the gate electrode, the path between the source and drain electrodes can be made to have higher or lower resistance. The gate voltage electrostatically changes the chemical potential inside the conduction channel, changing the size and shape of this path for the charge carriers. The conduction channel then accumulates or is depleted of charge carriers, which allows current to flow or be turned off respectively. Hence, changing the applied gate voltage allows the state of the transistor to be controlled.

In some cases, this conducting channel does not automatically exist within the transistor. This occurs in both $\mathrm{n}$ and $\mathrm{p}$-type enhancement mode transistors and requires a certain value of positive and negative voltages respectively to turn "on" these transistors. The voltage required to do this is known as the threshold voltage.

When the conducting channel does automatically exist, the gate is used to create a depletion zone in the conducting channel which increases the resistance of the pathway from source to drain electrodes. This allows the transistor to be turned "off". This is the case in both $\mathrm{n}$ and $\mathrm{p}$-type depletion-mode transistors.

\subsection{Spin transistor}

Spin field-effect transistors, or spinFETs, were initially proposed in 1990 by Datta and Das. Similar to electrical field-effect transistors, the spinFET consists of ferromagnetic source and detector electrodes and can include a gate [5]. The simplest geometry of a spinFET can be realised as two ferromagnetic electrodes that are connected by a nonmagnetic conducting channel (see figure 2.2). 


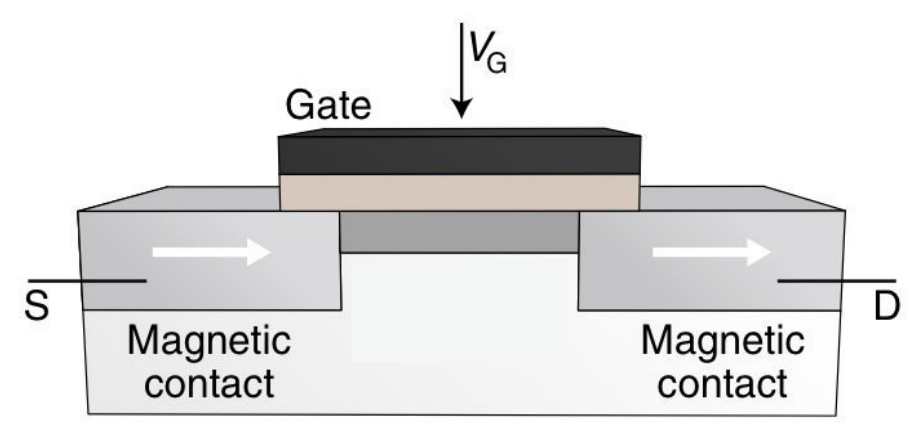

Figure 2.2: Altered spinFET schematic designed by Datta and Das [24]. The dark grey strip between the two magnetic contacts (or ferromagnetic electrodes) is the conducting channel. S and D indicate the ferromagnetic source and drain electrodes.

A conventional electronic transistor works to control the flow of charge current, but a spin transistor uses a 'spin polarised' current [25]. Charge current is a mixture of spin up and spin down electrons. Ferromagnetic materials have an imbalance of spin determined by the magnetisation of the material. When charge current is injected through a ferromagnetic electrode the current is 'spin polarised'. This means that the majority of electrons injected are of the same spin. At the interface where the charge current is injected, spin up and spin down electrons have different densities or electrochemical potentials (ECP) as a result of the injected spin polarised current [25].

Far away from the interface, charge current in a non-ferromagnetic material should have balanced spin densities. Therefore, along the conducting channel, the spin polarisation should decay away from the injection interface. This can also be described as the ECP of the two spin states equalising the further away from the interface they are. The distance it takes for the different ECP of the two spin states in a material to decay is characterised by the spin diffusion length [25]. This decay of spin is called a spin current [25].

If the conducting channel length is short enough, a polarised spin current will reach the second ferromagnetic electrode. If the second ferromagnetic electrode has the same magnetisation as the first, the spin current can enter the second ferromagnetic electrode with low resistance (see figure 2.3). If it has antiparallel magnetisation, the resistance is higher (see figure 2.3) [25]. These high and low resistance pathways are the two states of the spin transistor. 


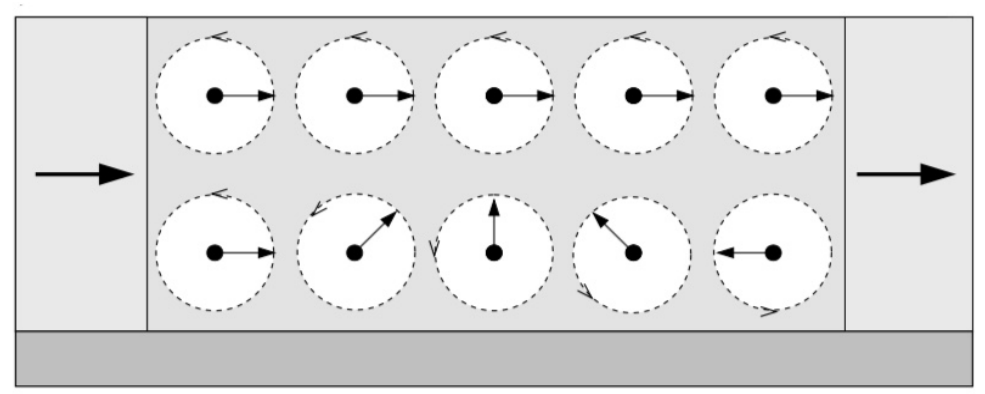

Figure 2.3: The top line of spins shows them reaching the second electrode with parallel alignment. The bottom shows the spins undergoing precession and reaching the second electrode with an anti-parallel alignment. Altered and sourced from [25].

To switch between the high and low resistance states in a spinFET, a magnetic or electric field can be applied to the conducting channel. This field causes the spins to precess, resulting in a change in spin polarisation at the second ferromagnetic electrode, causing the state of the transistor to change (see figure 2.3) [26]. Hence, the state of the system can be switched without changing the magnetisation of the ferromagnetic electrodes.

If the electrical current injected through the ferromagnetic electrode to the conducting channel does not flow through the conducting channel, there is no net flow of electrons between the two ferromagnetic electrodes. As there is no charge current in the conducting channel, the power dissipated is 0 as $P=I^{2} R$. Therefore utilising a spin current to control the two states of the transistor could result in a transistor with lower power consumption than a conventional electronic transistor.

Another potential power-saving capability of a spin transistor over a conventional electronic one is that the ferromagnetic electrodes hold their alignment regardless of what state the transistor is in. Even when switched entirely off, the ferromagnetic electrodes can preserve their magnetisation. As a spin transistor doesn't need to put in significant energy to maintain the state of the electrodes, it will predominantly dissipate power through only dynamic processes. [27] 


\subsection{The conductivity mismatch problem}

For a spin injection transistor to be realised, there needs to be a high spin injection efficiency from the ferromagnetic electrode to the non-magnetic conducting channel to provide a measurable spin voltage (see 2.6 for more details)[28].

To prevent a build-up of charge in the injector electrode, we want a continuous current density throughout the interface between the electrode and conducting channel. Continuous current density is required for high-efficiency injection of a spin current. As $\mathbf{J}=\sigma \mathbf{E}$, to produce continuous current, the conductivity of the two materials needs to match, assuming that they are both subject to the same electric field E.

The spin injection coefficient, $\gamma$, is a way of describing whether spin injection is likely to occur as a result of the relative conductivities of both materials [28]. The injection coefficient is dependent on the ratio $\gamma \propto \frac{\sigma_{N M}}{\sigma_{F M}}$ where $\sigma_{N M}$ and $\sigma_{F M}$ are the conductivities of the non-magnetic conducting channel and the ferromagnetic electrodes respectively [28]. The closer $\gamma$ is to 1 , the higher the spin injection efficiency. The smaller $\gamma$ is the lower the spin injection efficiency.

For a typical spin injection device fabricated with a semiconductor conducting channel and ferromagnetic metal electrodes where the ferromagnetic is cobalt [29] and the semiconductor doped gallium nitride [30] we find

$$
\begin{array}{r}
\rho_{\text {Cobalt }}=6 \times 10^{-6} \Omega \mathrm{cm} \\
\rho_{\text {GaN }}=0.02 \Omega \mathrm{cm}
\end{array}
$$

so

$$
\begin{aligned}
& \sigma_{\text {Cobalt }}=1.67 \times 10^{5} \mathrm{Scm}^{-1} \\
& \sigma_{G a N}=50 \mathrm{Scm}^{-1}
\end{aligned}
$$

the spin injection coefficient is proportional to 


$$
\gamma \propto \frac{\sigma_{\text {GaN }}}{\sigma_{\text {Cobalt }}}=\frac{50}{1.67 \times 10^{5}}=0.0003=0.3 \%
$$

Due to the conductivity mismatch between the electrodes and conducting channel the spin injection efficiency is very low.

\subsubsection{Solving the conductivity mismatch problem}

One solution to this problem is to use tunnel junctions between the ferromagnetic electrode and the semiconducting conducting channel [10]. Using a tunnel junction allows spin injection to be done by quantum mechanical tunnelling of electrons and is not dependent on the conductivities of the materials. By choosing a tunnel junction that has a higher probability of tunnelling for one spin than the other, spin injection efficiency can be higher [31]. A magnetic tunnel junction using a $G_{a} O_{x}$ barrier between iron electrodes and a magnesium oxide semiconducting layer was found to have a large magnetoresistance change of $92 \%$ [32]. $\mathrm{MgO}$ has also been utilised as a tunnel junction between iron electrodes and a silicon semiconducting layer, producing $90 \%$ spin injection polarisation [33]. A drawback of this method is the complexity of the tunnel junction structure resulting in a difficult fabrication procedure.

Another way of solving this problem would be to use materials for the electrodes and conducting channel that have similar conductivities. If a ferromagnetic semiconductor with tunable conductivity was used for the electrodes instead of a ferromagnetic metal, a device with $\sigma_{F M} \approx \sigma_{N M}$ such that $\frac{\sigma_{F M}}{\sigma_{N M}} \approx 1$ could be developed. This has been seen using the ferromagnetic semiconductor GaMnAs for the ferromagnetic electrodes, and the semiconductor GaAs conducting channel to make a vertical metaloxide-semiconductor field-effect transistor [34]. It's spin-dependent properties were investigated, and it was found that the source-drain current could be adjusted by up to $60 \%$. A tunnelling magnetoresistance change of $60 \%$ was also observed, indicating two clear states for the system. Although ferromagnetic semiconductors are rare, this project explores the use of a different one for the ferromagnetic electrodes in a spininjection device. 


\subsection{Rare-earth Nitrides}

Rare-earth Nitrides (RENs) are mostly ionic compounds, with a central lanthanide ion coordinated to six nitrogen ions (see figure 2.4). The lanthanide is usually in the $3+$ state and the nitrogens in the 3- state. The lanthanide, or rare-earth, has valence electrons in the $4 f$ shell [11]. Electrons in the $4 f$ shell have high angular momentum causing them to be highly localized. The RENs form crystals in the rock-salt structure (see figure 2.4). The nitrogen component of the RENs gives three electron carriers per site. Several of the RENs are ferromagnetic semiconductors, with ferromagnetic Curie temperatures up to $70 \mathrm{~K}$ [11]. Some RENs, including GdN, are also expected to have large spin polarisations [35].

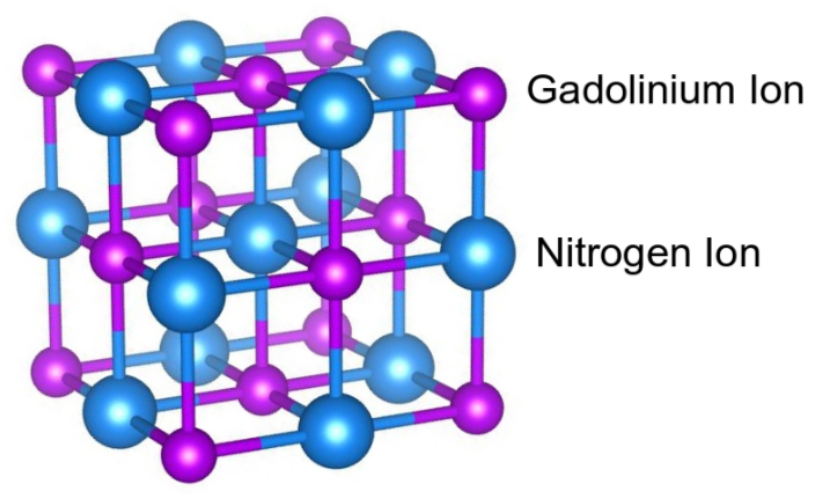

Figure 2.4: Rock-salt structure of a REN material, in this case Gadolinium nitride. Sourced from [36].

The electrical conduction properties are generally governed by the rare-earth $5 d$ electrons whilst the magnetic properties are governed by the highly localized rareearth $4 f$ electrons but are influenced by the number of nitrogen vacancies in the lattice [11]. Nitrogen vacancies are introduced to the REN crystals during the growth [37]. The conductivity of the RENs is adjustable by changing the nitrogen pressure at which the sample is grown. This is because at higher nitrogen pressures, fewer nitrogen vacancies can form in the REN lattice, so there is a lower carrier concentration relative to lower nitrogen pressures. As a result, RENs grown at higher nitrogen pressures have lower conductivity, compared to those grown at low nitrogen pressures.

In a previous study it was shown that the conductivity of GdN could also be tuned 
through magnesium doping across six orders of magnitude (see figure 2.5). The tunability of the conductivity of GdN means it is a good choice for an all semiconductor spin injection transistor, as the $\mathrm{GdN}$ can be grown to match that of the non-magnetic conducting channel material.

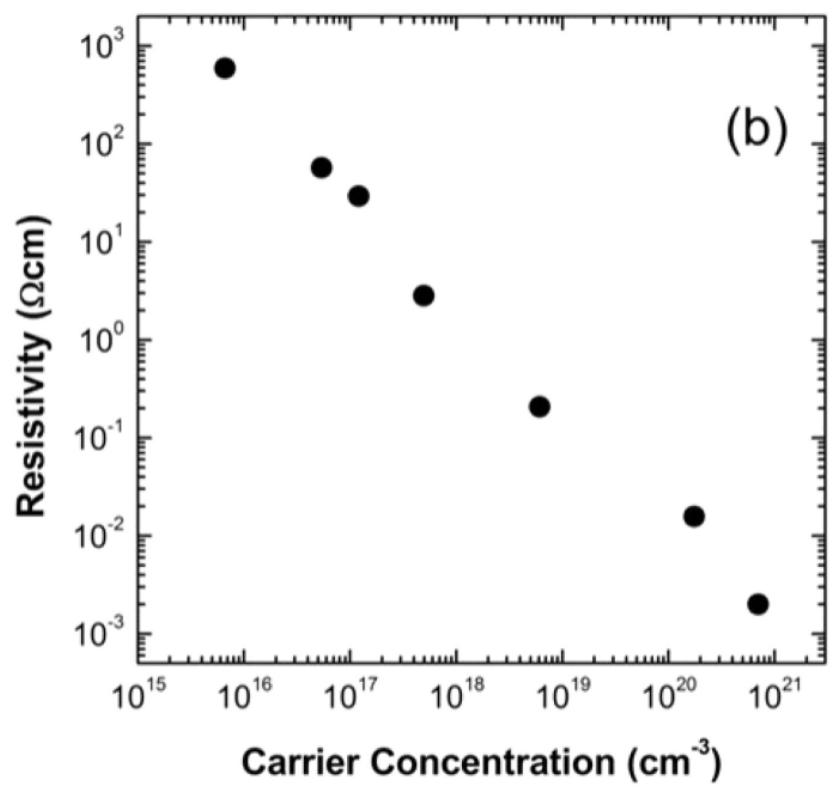

Figure 2.5: GdN thin films at room temperature [37] with $\mathrm{Mg}$ doping.

RENs are volatile and will rapidly oxidize when exposed to atmosphere [11]. There are two ways in which this can be minimised. One is to ensure that the RENs are grown in ultra-high vacuum chambers to avoid contaminants whilst depositing on substrates. The second is to ensure that before the samples are taken out of the chamber, a capping layer of protective material such as $\mathrm{Al}, \mathrm{AlN}$, or $\mathrm{GaN}$ is deposited on top of the REN. This allows the samples to be out in the atmosphere for measurements without imminent degradation.

In this project, GdN was used as the material for the spin injection electrodes as it has the highest observed onset Curie temperature of $70 \mathrm{~K}$ with high carrier concentration [38]. It is known to have a large spin polarisation [39], and is known to have controllable resistivity [37]. GdN is also seen to be compatible with growth on semiconductors such as GaN [40] [41], which could be utilised as a material for spin injection transistors at a commercial level. 
The coercive field of GdN can be tuned between $20 \mathrm{mT}$ and as low as $1 \mathrm{mT}$ [7]. It has small crystal directional dependence (anisotropy) which is what results in this small coercive field. What we get is a ferromagnet which has an easily flippable magnetic direction, making it ideal for a rewritable non-volatile device like a spin injection transistor. Any magnetic moment observed in GdN is a result of its half-filled $4 f$ orbitals. The magnetic moment is seen as $7 \mu_{B}$ from the $\mathrm{Gd}^{3+}$ ion when it is ferromagnetic [37]. $\mathrm{GdN}$ is also a narrow bandgap semiconductors with a down spin electron bandgap of $0.9 \mathrm{eV}$ and a $1.3 \mathrm{eV}$ bandgap for up spin electrons [42].

\subsection{Spin-injection measurement}

In other developments of spin injection devices, the spin injection measurement has been previously conducted using spectroscopy [43][44], non local electrical setups [45][46][26], and optically with Kerr microscopy [47][48]. The thesis is focused on electrical measurements of spin injection devices, as this is what will be needed eventually to incorporate a fully realised spinFET into semiconductor electronics.

Spin injection measurements are set up to be done using a non-local geometry allowing for a greater chance of measuring the spin-induced voltage than other configurations (see figure 2.6). The interpretation of electrical spin-transport measurements can be complicated by magnetoresistance in the electrodes, local Hall effects, and other extrinsic contributions to the signal [25]. A non-local measurement minimizes these background effects by placing a spin detection electrode outside the path of charge current.

As mentioned in 2.3, when the current is injected from the ferromagnetic electrode into the conducting channel, most of the injected electrons have a spin which is aligned with that of the injector ferromagnet (see figure 2.6). This produces a spin polarised charge current, as a well as a separate pure spin current. This gives an accumulation of spin in the conducting channel which can be described by the spin-dependent electrochemical potential (ECP), $\Delta_{\mu}=\mu_{u p}-\mu_{\text {down }}$. 


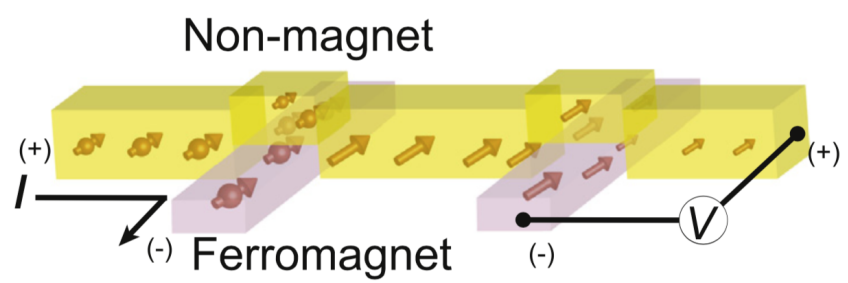

Figure 2.6: Non-local four point geometry measurement setup. Figure altered from [49]

When the polarised charge current is injected, the density of up-spin electrons in the non-magnetic conducting channel increases by shifting the electrochemical potential up by $\delta_{\mu_{N}}$. The density of down-spin electrons changes by $-\delta_{\mu_{N}}$ [25]. Consequently, the electrochemical potential undergoes a spin splitting of $2 \delta_{\mu_{N}}$. The spin current diffuses along the conducting channel towards the second ferromagnetic electrode where the $\Delta \mu$ manifests as a measurable voltage, $V_{2}$.

Although my devices were fabricated with a metallic channel, which is easier to make while working on patterning a spin injection device from the RENs, the theory for a spin injection device with a semiconductor channel illustrates clearly how the spin current signal behaves. The measured voltage at the second ferromagnetic electrode can be described by [50].

$$
\frac{V_{2}}{I}= \pm 2 R_{N} e^{-\frac{L}{\lambda_{N}}} \prod_{i=1}^{2}\left(\frac{P_{J} \frac{R_{i}}{R_{N}}}{1-P_{J}^{2}}+\frac{p_{F} \frac{R_{F}}{R_{N}}}{1-p_{F}^{2}}\right) \times\left[\prod_{i=1}^{2}\left(1+\frac{2 \frac{R_{i}}{R_{N}}}{1-P_{J}^{2}}+\frac{2 \frac{R_{F}}{R_{N}}}{1-p_{F}^{2}}\right)-e^{\frac{-2 L}{\lambda_{N}}}\right]^{-1}
$$

With $P_{J}$ as the interfacial current polarisation, $p_{F}$ as the current polarisation of the ferromagnet, and $R_{F}$ and $R_{N}$ the resistances of the ferromagnet and semiconductor cross-sections respectively. $\Pi$ is the Pi function and works by multiplying the relevant terms up the maximum integer for $i$. The integer $i$ represents the two electrodes in the system. The cross-section between the two materials is the area of overlap at the junction. We can also see that the voltage can be either positive or negative, and this corresponds to the high and low resistance states resulting from the antiparallel and 
parallel magnetic alignments of the ferromagnets [50].

To gain information about spin transport within the spin injection device, we have to determine how the behaviour of the spins depends on the relative states of the ferromagnetic electrodes. This signal can be thought about as either the resistance change

$$
R_{s}=\frac{V_{s}}{I}
$$

or the change in voltage,

$$
V_{s}=V_{2}^{P}-V_{2}^{A P}=2\left|V_{2}\right|
$$

when the alignment of the ferromagnetic electrodes changes from parallel to antiparallel.

To determine a characteristic equation for my devices we start by substituting equation 2.4 into equation 2.6 and expand the $\prod$ function.

Applying the $\prod$ function to the first term we find

$$
\left(\frac{2\left|V_{2}\right|}{I}\right)_{\text {numerator }}=4 R_{N} e^{-\frac{L}{\lambda_{N}}}\left(\frac{P_{J} \frac{R_{1}}{R_{N}}}{1-P_{J}^{2}}+\frac{p_{F} \frac{R_{F}}{R_{N}}}{1-p_{F}^{2}}\right)\left(\frac{P_{J} \frac{R_{2}}{R_{N}}}{1-P_{J}^{2}}+\frac{p_{F} \frac{R_{F}}{R_{N}}}{1-p_{F}^{2}}\right)
$$

Assuming the electrochemical potentials between the semiconductor and ferromagnetic layers are continuous, the interfacial conductance becomes $G_{i} \rightarrow \infty$ [50]. This is referred to as having transparent contacts. The interfacial current polarisation is proportional to $G_{i}$ as $P_{J}=\frac{\left|G_{i}^{\uparrow}-G_{i}^{\downarrow}\right|}{G_{i}}$. Therefore $P_{J} \rightarrow 0$. Consequently the first two terms in each bracket in (2.7) cancel to give 


$$
\begin{aligned}
\left(\frac{2\left|V_{2}\right|}{I}\right)_{\text {numerator }} & =4 R_{N} e^{-\frac{L}{\lambda_{N}}}\left(\frac{p_{F} \frac{R_{F}}{R_{N}}}{1-p_{F}^{2}}\right)\left(\frac{p_{F} \frac{R_{F}}{R_{N}}}{1-p_{F}^{2}}\right) \\
& =4 R_{N} e^{-\frac{L}{\lambda_{N}}}\left(\frac{p_{F} \frac{R_{F}}{R_{N}}}{1-p_{F}^{2}}\right)^{2}
\end{aligned}
$$

Applying the $\prod$ functions to the second term we find that

$$
\left(\frac{2\left|V_{2}\right|}{I}\right)_{\text {denominator }}=\left(1+\frac{2 \frac{R_{1}}{R_{N}}}{1-P_{J}^{2}}+\frac{2 \frac{R_{F}}{R_{N}}}{1-p_{F}^{2}}\right)\left(1+\frac{2 \frac{R_{2}}{R_{N}}}{1-P_{J}^{2}}+\frac{2 \frac{R_{F}}{R_{N}}}{1-p_{F}^{2}}\right)-e^{\frac{-2 L}{\lambda_{N}}}
$$

We can once again assume that $P_{J} \rightarrow 0$. Following on from the assumption of transparent contacts, we know that $R_{1}, R_{2} \ll R_{N}$ [50]. Hence we can cancel the second term in each bracket and then expand to find

$$
\begin{aligned}
\left(\frac{2\left|V_{2}\right|}{I}\right)_{\text {denominator }} & =\left(1+\frac{2 \frac{R_{F}}{R_{N}}}{1-p_{F}^{2}}\right)\left(1+\frac{2 \frac{R_{F}}{R_{N}}}{1-p_{F}^{2}}\right)-e^{\frac{-2 L}{\lambda_{N}}} \\
& =1+\frac{4 R_{F}}{R_{N}\left(1-p_{F}^{2}\right)}+\frac{4 R_{F}^{2}}{R_{N}^{2}\left(1-p_{F}^{2}\right)}-e^{\frac{-2 L}{\lambda_{N}}}
\end{aligned}
$$

Assuming that the ferromagnetic electrodes are made of a more conductive material than the conducting channel, $R_{F} \simeq 0.01 R_{N}$. This is an assumption based on metallic ferromagnetic electrodes and a more resistive conducting channel. Applying this, the second order term is negligible producing

$$
\left(\frac{2\left|V_{2}\right|}{I}\right)_{\text {denominator }}=1+\frac{4 R_{F}}{R_{N}\left(1-p_{F}^{2}\right)}-e^{\frac{-2 L}{\lambda_{N}}}
$$


Combining both parts we are able to find that

$$
R_{s}=\frac{2\left|V_{2}\right|}{I}=\frac{4 R_{F}^{2}}{R_{N}} \frac{\left(\frac{p_{F}}{1-p_{F}^{2}}\right)^{2} e^{\frac{-L}{\lambda_{N}}}}{\left(1+\frac{4 R_{F}}{R_{N}\left(1-p_{F}^{2}\right)}\right)-e^{\frac{-2 L}{\lambda_{N}}}}
$$

The voltage, and corresponding resistance measured for each of the two states is dependent on the relative resistance of two materials.

Considering the cases where two materials used had similar resistances with $R_{F} \simeq$ $R_{N}$, or the ferromagnetic material was more resistive than the conducting channel, $R_{F} \simeq 10 R_{N}$. In these cases, the assumptions applied to find equations 2.10 and 2.11 do not hold, and the final equation for the resistance of the system will appear closer to equations 2.8 and 2.9. The dependence of the measured voltage on the relative resistances of the materials used would change, but the dependence on the magnetisation states for the electrodes as well as the exponential dependence on distance does not change. 


\section{Chapter 3}

\section{Experimental Methods}

In this chapter the methods and processes of developing my spin injection device are described across three sections. The first section explains the process of sample preparation, the second deals with the experimental techniques used to characterise my devices, and the final section outlines the experimental setup developed for spin injection and other low temperature measurements.

\subsection{Sample fabrication}

The process of growing the samples used in this research thesis required two main steps. Thin-film microfabrication techniques such as photolithography and thermal evaporation were used, as well as growth of GdN under ultra-high vacuum. The equipment used was based in the clean room of the School of Chemical and Physical Sciences at VUW. My device consists of four copper contact pads, connected to two GdN electrodes, that in turn are connected via a copper conducting channel (see figure 3.1b). Samples were all patterned and grown on 2-inch wafers of Si with 300nm of $\mathrm{SiO}_{2}$. GdN readily forms a silicate [11], so the $\mathrm{SiO}_{2}$ formed a barrier to stop this occurring. Each wafer had multiple devices patterned onto it, allowing for devices with different dimensions to be fabricated simultaneously (see figure 3.1a). The distance between the ferromagnets was varied, as well as the width of the electrodes. The distances were chosen to be $2 \mu \mathrm{m}, 5 \mu \mathrm{m}$, and $10 \mu \mathrm{m}$. The smallest distance of $2 \mu \mathrm{m}$ was chosen as this is the lower limit of our patterning. It was important to ensure that devices were made so that the distance between the electrodes was less than $\sim 5$ times 
the spin diffusion length of copper at $10 K, 1000 \mathrm{~nm}$ [51]. This is because as seen in 2.12, the signal from the spin injection decays exponentially with distance from the point of injection. If the distance between the electrodes is too great, the spin signal is unlikely to be measurable. The distance between the electrodes ranged between $2 \mu \mathrm{m}, 5 \mu \mathrm{m}$, and $10 \mu \mathrm{m}$ as well, to provide variation in the area of spin injection possible. The shapes of the ferromagnetic electrodes differ so that the electrodes switch magnetisation direction at different applied magnetic fields, allowing for anti-parallel alignment of the electrodes to be acheived.

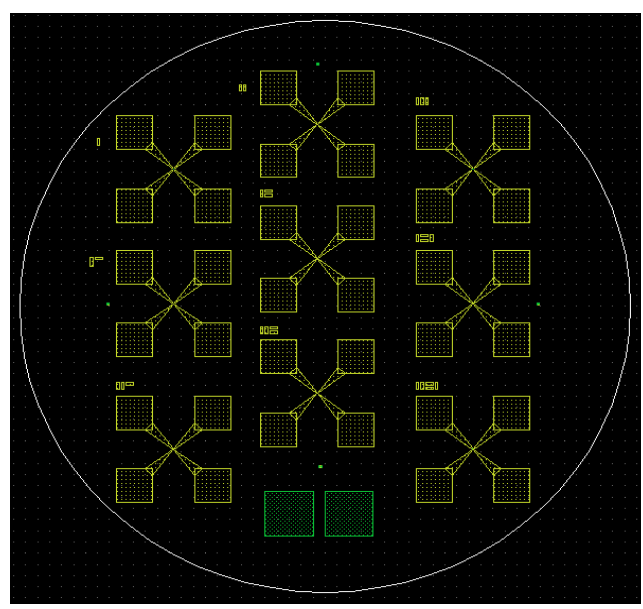

(a) Mask design for full wafer

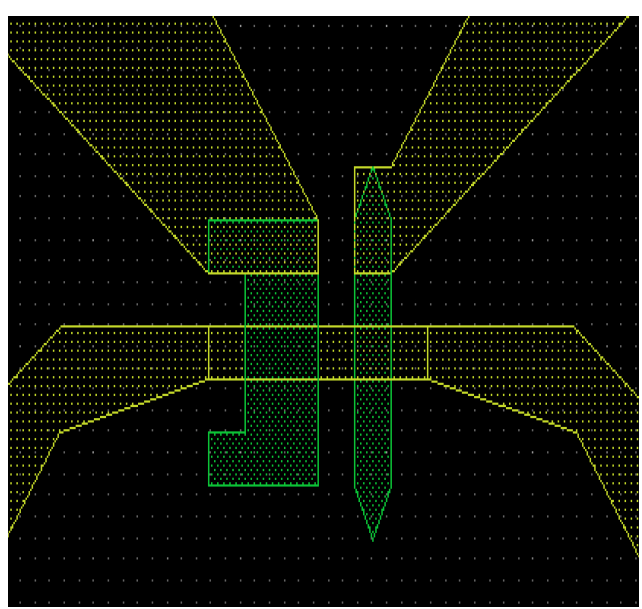

(b) Design of one device

Figure 3.1: My photomasks were designed on LayoutEditor [52]. Yellow features represent where the copper would be deposited, and green features represent the GdN. The white circle in (a) shows the outline of the wafer.

\subsubsection{Photolithography}

Photolithography (also known as optical lithography) is a common process used to pattern thin films and/or substrates in microfabrication. Lithography itself refers to the transfer of shapes on a mask to a smooth surface. In photolithography, light is used to transfer a pattern from a photomask by interacting with the photosensitive photoresist on the substrate. These photomasks are physical masks and allow light through in select areas. The photomasks used for my devices were produced at the Nanofabrication Laboratory of University of Canterbury. During this step, the photoresist covered substrate is exposed to UV light and is photopolymerized. This allows the photoresist to 
be soluble when exposed to photodeveloper. When submerged in photodeveloper, the polymerized photoresist is removed, resulting in the desired pattern on the substrate.

The first step was patterning the substrate to allow for the deposition of copper to form the contacts and conducting channel of my devices. There are four positional alignment markers patterned into the substrate; two at either end of the middle column of devices, and two on either side of the three columns of devices. These alignment markers consist of nine $20 \times 20 \mu \mathrm{m}$ squares in a checkerboard pattern (see figure 3.2a). The alignment marks make it easy to determine if there is any displacement or rotational misalignment in the second step of photolithography. Each device is numbered for easy identification after growth. A single device with contacts can fit on a $10 \times 10 \mathrm{~mm}$ chip, with each device measuring $9.5 \times 9.5 \mathrm{~mm}$ at the outer contact edge. All contact pads have dimensions $3 \times 3 \mathrm{~mm}$, and have a separation of $3.5 \mathrm{~mm}$ to allow for easy manual connection of wires for electrical measurements (see figure 3.2b). Square areas at the bottom of the full substrates can be used for any structural or magnetic characterisations, as well as aiding in alignment (see figure 3.1a). Measurements only required one device at a time to be in the PPMS, so all devices are a suitable distance apart to ensure efficient substrate cleaving.

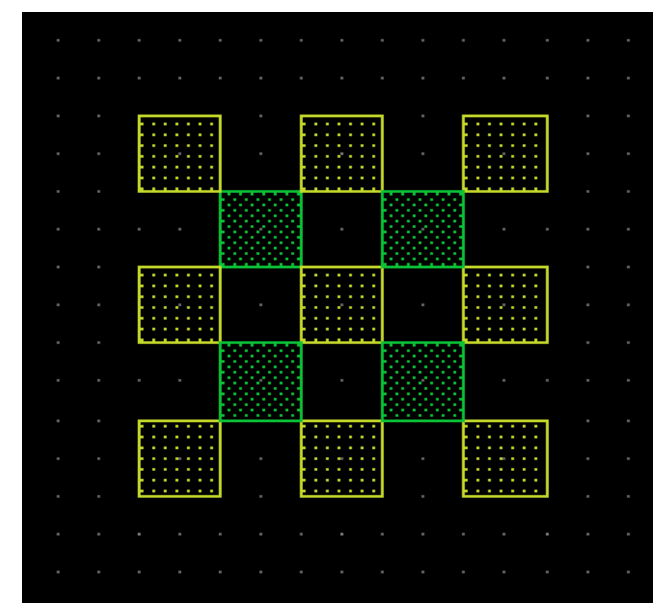

(a) Design of one set of alignment marks

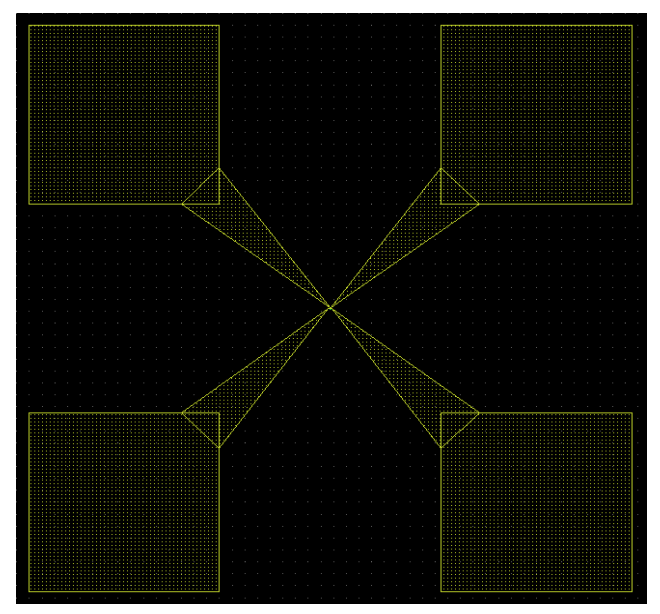

(b) One full device design with contact pads

Figure 3.2: As with figure 3.1, yellow features represent where the copper would be deposited, and green features represent the GdN. 


\section{Photolithography recipe}

1. Spin coat sample with AZ5214E at $4000 \mathrm{rpm}$ for $60 \mathrm{~s}$

2. Soft bake coated sample for 1 minute at $95^{\circ} \mathrm{C}$. This is used to evaporate the coating solvent, and increase the density of the resist after spin coating.

3. Expose the entire wafer to UV light for $0.25 \mathrm{~s}$

4. Hard bake sample for 2 minutes at $110^{\circ} \mathrm{C}$. This is to stabilize and harden the photoresist before processing steps that use the photomask. It also removes any traces of the solvent or excess developer and improves adhesion of the photoresist to the wafer surface.

5. UV expose for $12 \mathrm{~s}$ through aligned patterned photomask.

6. Develop baked sample in 4:1 deionised water and 351:B photodeveloper solution for 20s

7. Double rinse wafer in deionised water and blow dry with nitrogen

8. Store sample in a container for up to two months. Does not have to be in vacuum unless sample already contains materials sensitive to air

This process was developed by Dr Leo Browning for his PhD [53].

It is important to check the substrate under the microscope at each step to ensure development is fully realised, and there is no residual photoresist or developer. This is important for clarity of the pattern, and to ensure no contamination from this process.

The photoresist AZ5214E was chosen as it consistently gave clean edge definition on the devices. Sample steps 3 and 5 were done on a Karl Suss MJB3 Mask Aligner.

\subsubsection{Contact pad deposition}

Using the Angstrom engineering evaporator I evaporated copper and chrome onto a sample after the first step of photolithography was complete. Both the copper and 
chrome to make the contacts and conducting channel were thermally evaporated. Copper was chosen as to ensure maximum signal at the second ferromagnet, the conducting channel material needed to have a long spin diffusion length [25]. Moreover, the conducting channel material needs to be non-magnetic to not disrupt spin injection. Copper is also non-toxic and cheap so was appropriate as the material for the contacts. Chrome was evaporated to provide an adhesion layer so the copper would stick to the substrate and not be removed during lift-off (see figure 3.3).

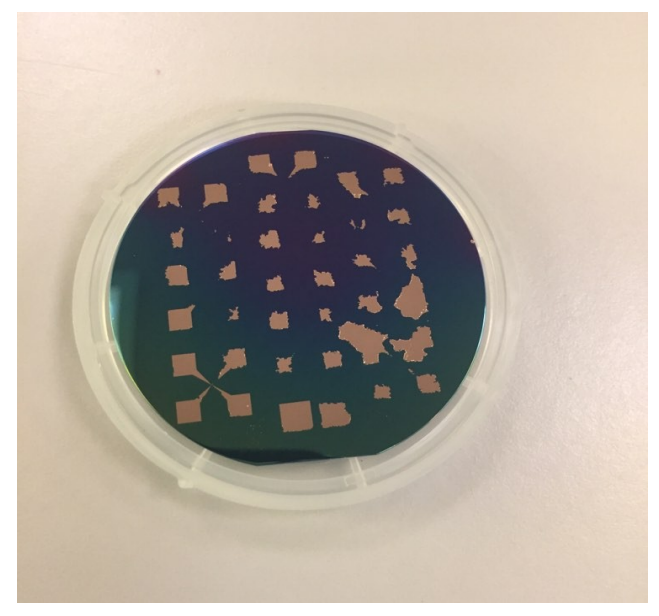

(a) Without chrome layer

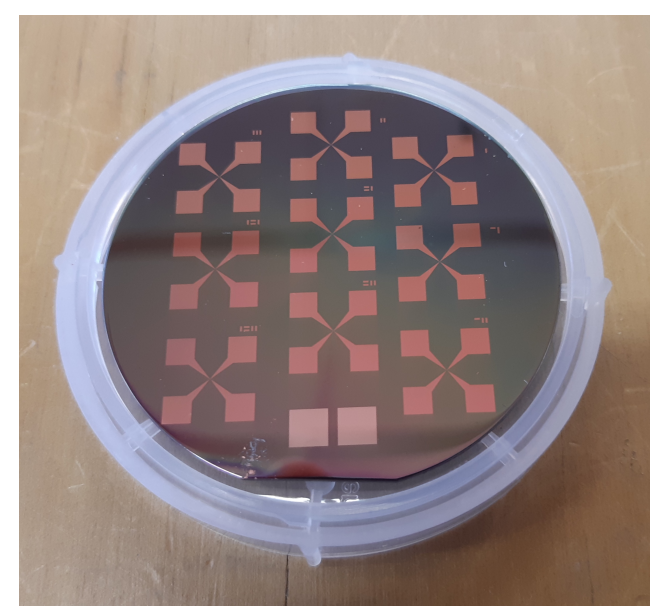

(b) With chrome layer.

Figure 3.3: Full wafers after copper deposition with and without adhesive chrome layer under copper. The wafer without chrome has a layer of resist from the photolithographic patterning of the GdN electrodes resulting in the difference in colour.

Wafers were taped photoresist side up onto the rotating holder and placed into the evaporation chamber upside down. The evaporator was left to pump down overnight to achieve the best possible vacuum. This is required, as while evaporating the pressure inside the chamber increases, and if it exceeds $5 \times 10^{-5}$ Torr the evaporator will trigger a safety interlock and automatically shut down. This low-pressure environment decreases the number of contaminants that can end up deposited on the sample during evaporation to produce highly conductive contacts. Ideally, the base pressure when starting the evaporation is $1 \times 10^{-6}$ Torr.

The metal is evaporated from the bottom of the evaporator, while the wafer is on the underside of the rotating platform. The holder rotates during the evaporation re- 
sulting in a uniform deposition of the evaporated material. A shutter within the evaporation chamber allows for deposition to only occur when the material is evaporating at the desired rate and is also used to stop deposition when required. First, the 5nm of chrome was evaporated, and then 50nm of copper with the entire thermal evaporation process taking just under an hour. After the evaporation was complete, the evaporator was stopped and vented to atmosphere so the samples could be removed for the next step of device fabrication.

Lift-off was done to remove any unnecessary material after deposition. The samples were left in acetone, and any part of the wafer not exposed to UV light during the photolithography process "lifts-off" the wafer, leaving only the patterned areas with the $\mathrm{Cr} / \mathrm{Cu}$ layer (See figure 3.3).

\section{Lift-off recipe}

1. Submerge wafer in glass container with acetone for 1-2 hours.

2. Place in a new glass container with acetone and sonicate for 1 minute.

3. Rinse with isopropyl alcohol and then deionised water.

4. Dry with nitrogen.

\subsubsection{Patterning of GdN electrodes}

After the wafer has gone through liftoff to remove unwanted copper, the substrate then goes through the second step of photolithography to pattern the ferromagnetic GdN electrodes. The second mask also has four alignment markers. This time the four corner markers consist of a set of four 20x20 $\mathrm{m}$ squares which fit inside the previously patterned nine copper alignment squares (see figure 3.2a). After photolithography has been completed, the GdN is grown along with the capping layer to protect the GdN from oxidising in atmospheric conditions. Both $\mathrm{GaN}$ and $\mathrm{AlN}$ were chosen as capping layers due to previous success in preventing oxidation of the GdN samples, and their ability to be seamlessly deposited on top of the GdN layer [54][11]. 
Ideally, the ferromagnets should be as close to pure GdN as possible to avoid oxidation and other negative impacts of contaminants. This ensures that our devices will have the required electrical and magnetic properties to establish spin injection and detection. To do this, the $\mathrm{GdN}$ and capping layer were grown in ultra-high vacuum (UHV) chambers. These chambers are made of stainless steel and the pressure can be kept below $1 \times 10^{-8}$ mbar $\left(1 \times 10^{-9}\right.$ Torr $)$. Inside these chambers we can grow both the GdN and the capping layer via physical vapour deposition (PVD), and ensure that the GdN does not come into direct contact with the atmosphere until after the protective capping layer is deposited.

After the wafer is loaded into the UHV chamber, the Gd held in a crucible is heated with an electron gun until it evaporates. At this point, it is exposed to an atmosphere of nitrogen at a partial pressure around $10^{-5}$ mbar to $10^{-4}$ mbar. The Gd catalytically cracks the molecular nitrogen to form GdN. This forms on the substrate (as well as surrounding chamber walls) to be the thin film growth (see figure 3.4). Once the GdN is at the desired thickness, the capping layer is deposited. After this, the wafer is removed from the UHV chamber and undergoes liftoff again to remove the excess GdN from the wafer. It is then stored in a desiccator to limit atmosphere exposure. Refer to chapter 4 for specific growth details for the GdN and capping layer.

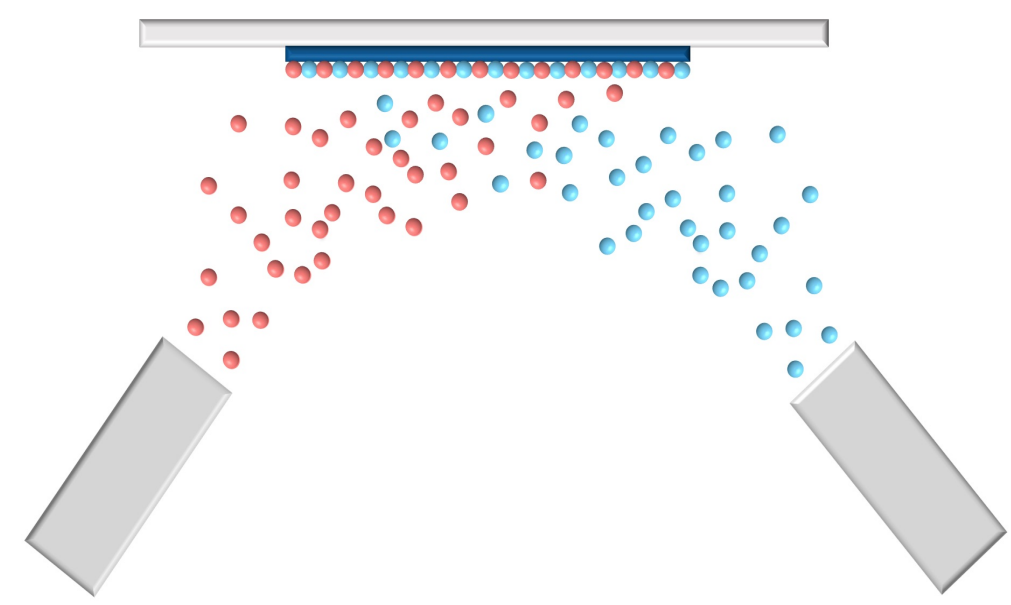

Figure 3.4: Growth of GdN, with e-gun heated Gd (bottom right), and $N_{2}$ (bottom left) onto patterned silicon wafer (top). 


\subsection{Characterisation Equipment}

X-ray diffraction (XRD) was used to determine the materials present within my samples. The principles of XRD can be found in Introduction to Solid State Physics 8th Edition by Charles Kittel [55]. The X-ray diffractometer used was a Bruker D8 Advance system using a cobalt $K_{\alpha}$ X-ray source with a wavelength of $1.789 \AA$ based at the Gracefield campus of Callaghan Innovation. A $2 \Theta$ sweep from $20^{\circ}$ to $90^{\circ}$ was done to determine the presence and orientation of the GdN crystals grown (see figure 3.5). As the width of the GdN electrodes was less than 10um wide, XRD data was not collected using a spin injection device (see figure 3.1a).

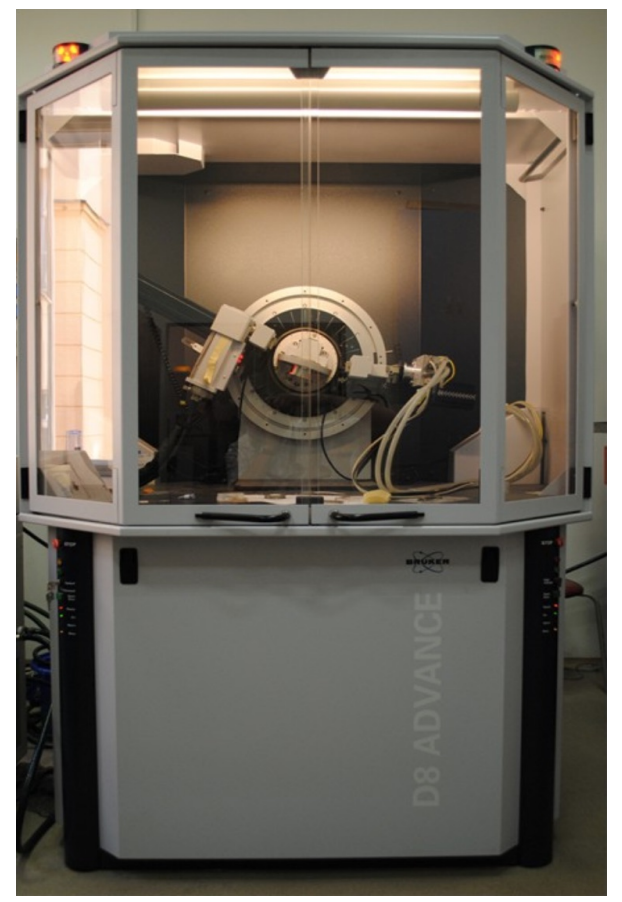

Figure 3.5: X-ray diffractometer similar to that at Callaghan Innovation used to help characterise my devices. Sourced from [56].

The Quantum Design Physical Properties Measurement System (PPMS) based at the Robinson Research Institute was also used for characterisation (see figure 3.6). Samples can be exposed to magnetic fields of up to $\pm 9 \mathrm{~T}$, and temperatures between 1.9 and $400 \mathrm{~K}$. For my samples, it was used to characterise the REN through measuring the resistance. Just checking the resistance allowed us to determine if a good electrical contact had been made. 


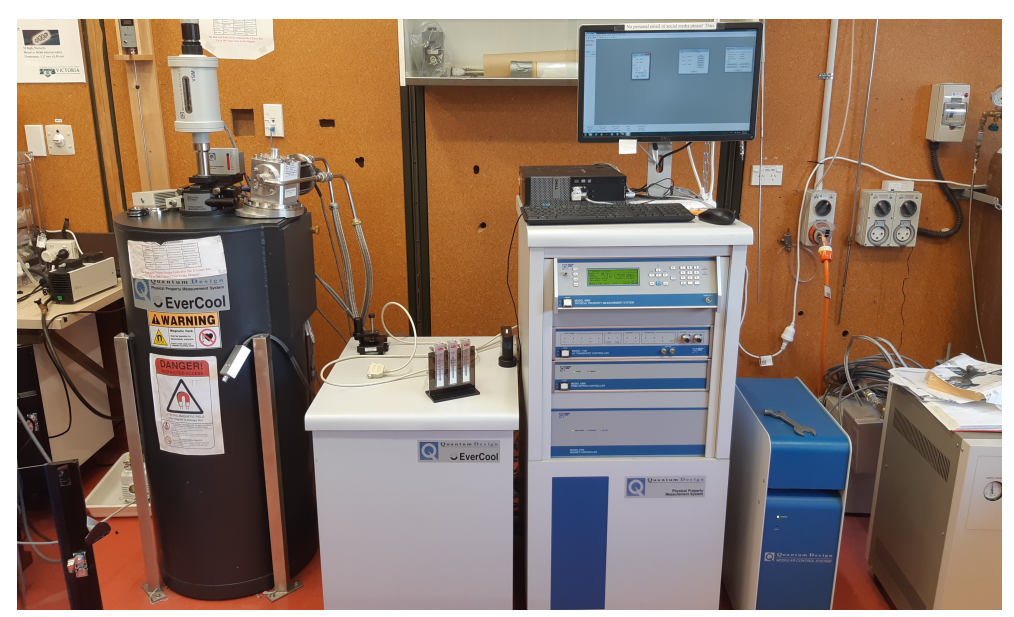

Figure 3.6: PPMS used for characterisation.

By cooling down the sample, it was used to determine the resistance as a function of temperature. This told us about the conductivity of the GdN. If the resistance drops at a lower temperature it is metal-like, and if the resistance were to increase at a lower temperature it is semiconductor-like. My devices were put on the horizontal rotator, and placed in the PPMS (see figure 3.7). The horizontal rotator enables a transport sample to be rotated over a full $360^{\circ}$ in the presence of an applied magnetic field.

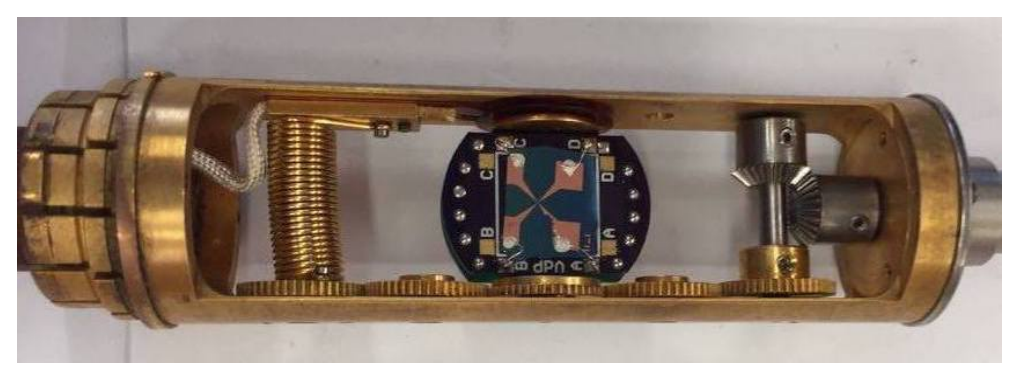

Figure 3.7: PPMS puck with wire connections made, placed in the horizontal rotator.

My sample was attached to the standard PPMS rotator puck using GE varnish. The sample was then electrically connected to the puck using annealed silver wire of diameter $0.125 \mathrm{~mm}$. The silver wire purchased from Goodfellow was attached to the puck using solder, and then to copper contact pads of the sample with RS PRO silver conductive paint. To ensure the paint fully dried and would have a reliable electrical connection, the sample holder with the sample was put in an oven at $120^{\circ} \mathrm{C}$ 
for 30 minutes. Once the sample was attached and the wire connections had been made, the puck was plugged into the horizontal rotator that was put in the PPMS for measurement (see figure 3.7).

\subsection{Spin-injection measurement system}

A spin-injection measurement system was set up during this project for electrical spintransport measurements for future developed spin-injection devices. This was greatly supported by the Shiraishi Laboratory at the Department of Electronic Science and Engineering, Kyoto University, whose collaboration helped streamline our physical system set up, but also contributed the LabVIEW program used to run our equipment.

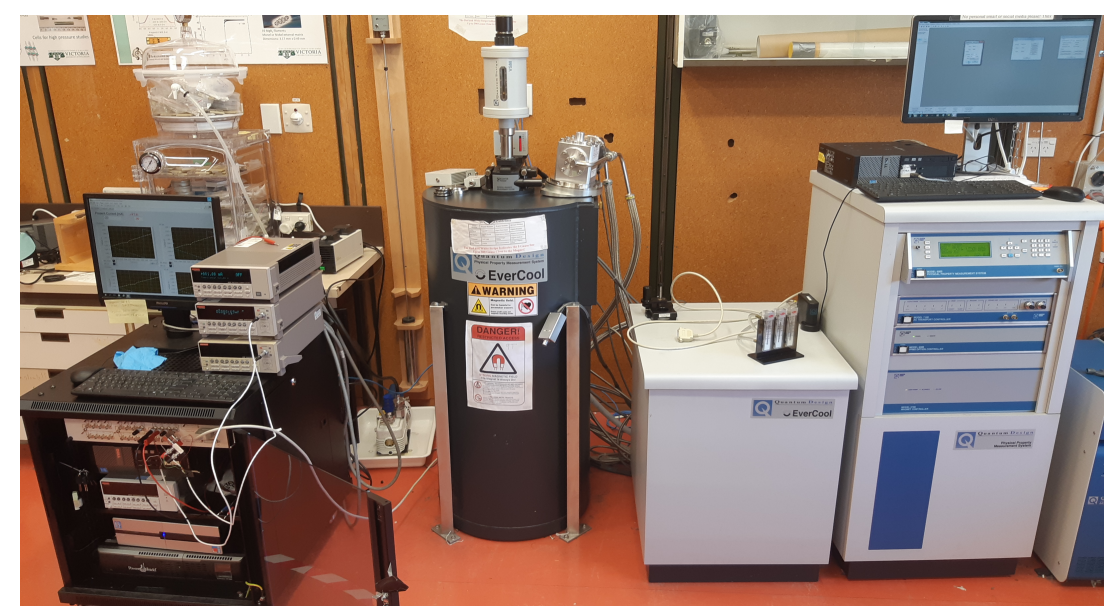

Figure 3.8: Full setup of spin-injection measurement system.

There are five main parts of the spin-injection measurement system (see figure 3.8). The first is the current source. The current is produced by a Keithley 6220 precision current source (see figure 3.9a). This, when connected to the rest of the equipment, is controlled by the LabVIEW program to input current through to the sample in the PPMS. The PPMS acts as the measurement chamber and is also controlled by the LabVIEW program. It allows the sample to be exposed to the variations in temperature and magnetic field during an experiment. As detailed in chapter 3.2, the sample is put onto the puck and horizontal rotator and placed in the PPMS. This connects the electrical contacts from the sample to the outside of the chamber, where it can be eventually connected to the current and voltmeters. When the experiment is running, the voltage 
is measured by one of two Keithley 2182A nanovoltmeters (see figure 3.9a).

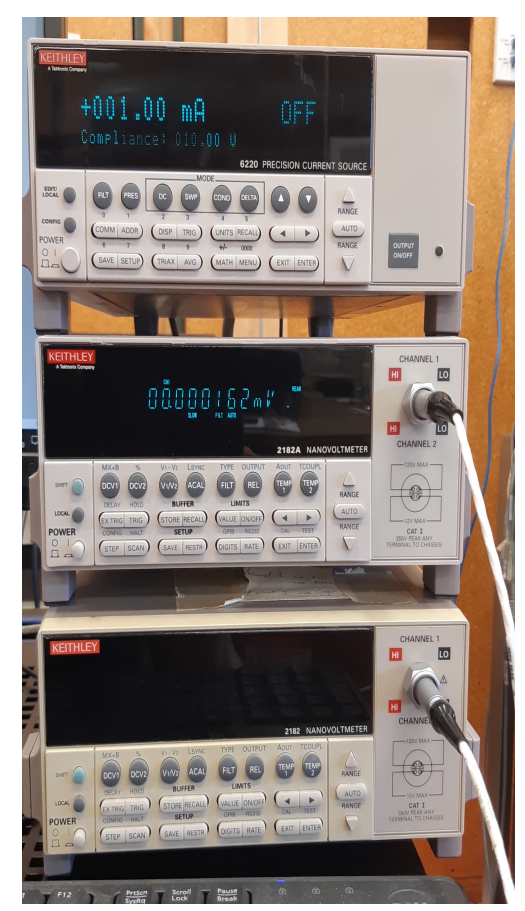

(a) Current source (top) and Nanovoltmeters

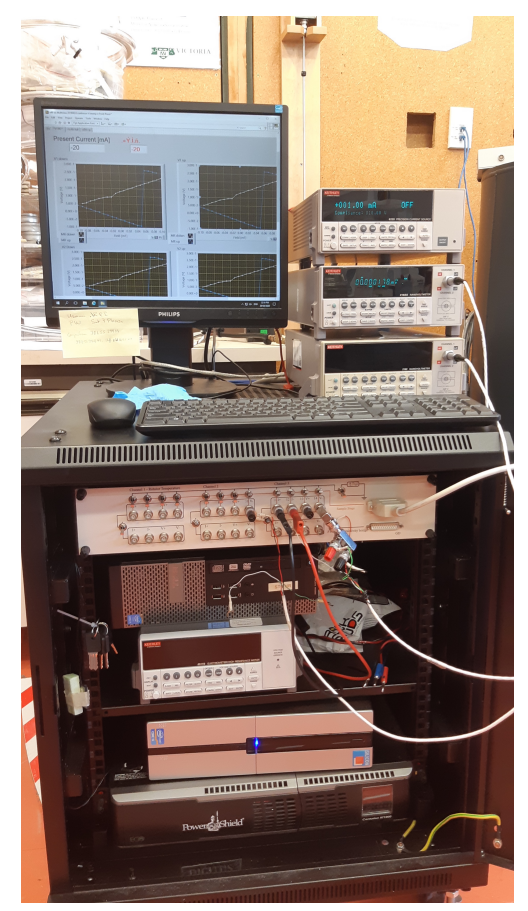

(b) LabVIEW computer with breakout box, current source, and voltmeters

Figure 3.9

The next part of the set up is the breakout box. The breakout box allows us to control current flow through the device and allows the configuration of the current flow and voltage measurements to be easily rearranged. My breakout box design (see figures 3.10b and 3.10a) was modelled off that at Kyoto University in the Shiraishi Laboratory. It has a $10 k \Omega$ resistor in series with the connection to the PPMS, as well as being connected to two $20 \mathrm{k} \Omega$ and a $100 \Omega$ resistor located between the current source and breakout box. These resistors stop the current from instantaneously going through the sample in the PPMS, as well as slowly dissipating any built up charge or voltage difference between the connections, preventing damage to the device from an overload of current. Each of the ports on the front of the breakout box is connected to a different spoke on the PPMS cable (see figure 3.10a). This means that in order to change the configuration of the measurement, the device does not need to be rewired, rather only the cables on the front of the breakout box need to be changed. 


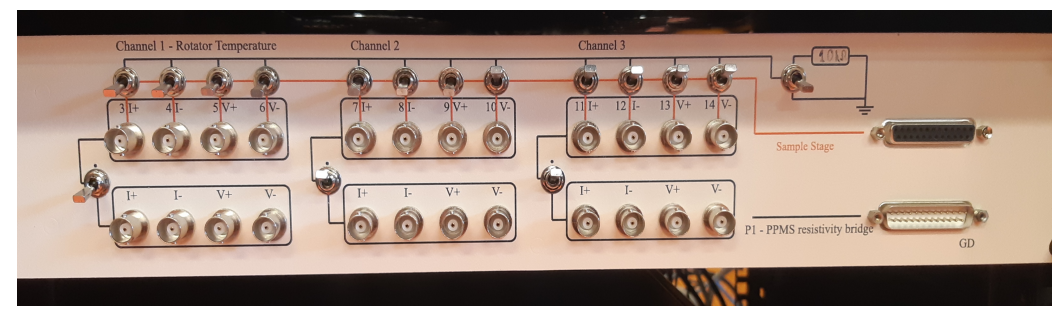

(a) Front panel of breakout box

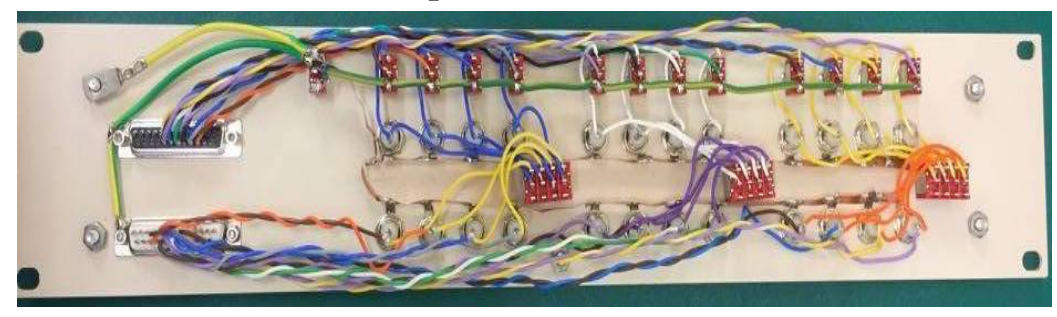

(b) Internal wiring of breakout box

Figure 3.10: Breakout box

The final part is the LabVIEW program which controls all equipment used in the experimental setup. LabVIEW is a visual programming software from National Instruments, useful for controlling lab equipment [57]. Written into the code is the electrical current chosen to go through the device, the temperature(s) the devices are exposed to, and the rate of magnetic field sweeping if applicable. The program reads the voltages measured using the nanovoltmeters and writes it into allocated files. The program allows the temperature, applied magnetic field, applied current, and the intervals of the temperature and field to be changed as required.

The measurements require the following steps to be done in this exact order so as to avoid overloading the device being measured with the current.

1. First the sample is connected to the puck, put on the horizontal rotator and put into the PPMS (see chapter 3.2).

2. On the computor that directly conrols the PPMS, a program called MutliVu is first set up to allow connection over the local network to the computor that controls the LabVIEW program. The LabVIEW programme will control the PPMS via MultiVu.

3. Next, the voltmeters and current source are connected to the breakout box in 
the desired configuration for the chosen measurement. Ensure the PPMS is connected to the breakout box. Check that all connections on the breakout box are in the "off" position aside from the required connections.

4. Ensure the current source is connected so that the applied current must travel through each $10 k \Omega, 20 k \Omega$, and $100 \Omega$ resistors before going to the PPMS. Check that the variable $20 k \Omega$ resistors are at their highest value, with the knobs turned fully to the right.

5. Set the current on the current source to $0 \mu A$ and switch the current on. Slowly start dialling down the resistances between the current source and PPMS. First the $20 k \Omega$ resistors, then the $100 \Omega$ resistor, and finally the $10 k \Omega$ resistor.

6. Open LabVIEW program, and start the measurement. This will begin the cooling down of the sample.

7. Once the measurement is complete, ensure the current applied is $0 A$. Then turn up the resistance for each resistor between the current source and PPMS, and switch the breakout box's connections to be shorted. Next remove the sample from the PPMS, and place in a desiccator for storage. 


\section{Chapter 4}

\section{Results and Discussion}

In this chapter the results of my project and a discussion of them are presented and split into two sections. The first section details the fabrication of the GdN based spin injection devices, while the second deals with the tests done on the fabricated devices to determine their integrity.

\subsection{Fabrication}

Three wafers resulted in successfully fabricated spin injection devices. These fabrications were not focused on matching the conductivity of the GdN to the copper, rather the goal was to determine if the device design and lithography based fabrication for making spin injection devices using GdN apply to a real-life situation, and what limitations the fabrication process is subject to.

\subsubsection{GdN growth results}

Of these three wafers, two GdN depositions were grown to be insulating, and the other was grown to be more conductive (see table 4.1 for growth conditions). All three wafers were grown in the Thermionics UHV system of the spintronics group of SCPS at VUW by Dr Denis Lefebvre and Dr Franck Natali. The two insulating samples had two different insulating capping layers: AlN and GaN. These samples will be referred to as $\mathrm{W} 1$ and W2 respectively. When these insulating capping layers were used, the entire 
GdN layer was transparent, and allowed for the overlap between the copper and GdN to be seen (see figure 4.1 ).

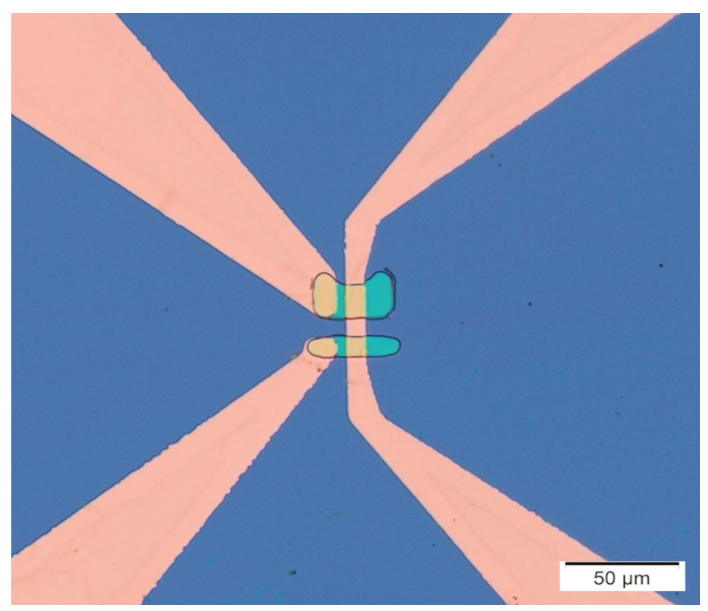

Figure 4.1: Microscope photo of sample W2 that was capped with insulating GaN. This device had a distance of $10 \mu \mathrm{m}$ between electrodes. The overlap of the electrodes and the conducting channel and contacts can be easily seen due to the transparency of the GdN and GaN.

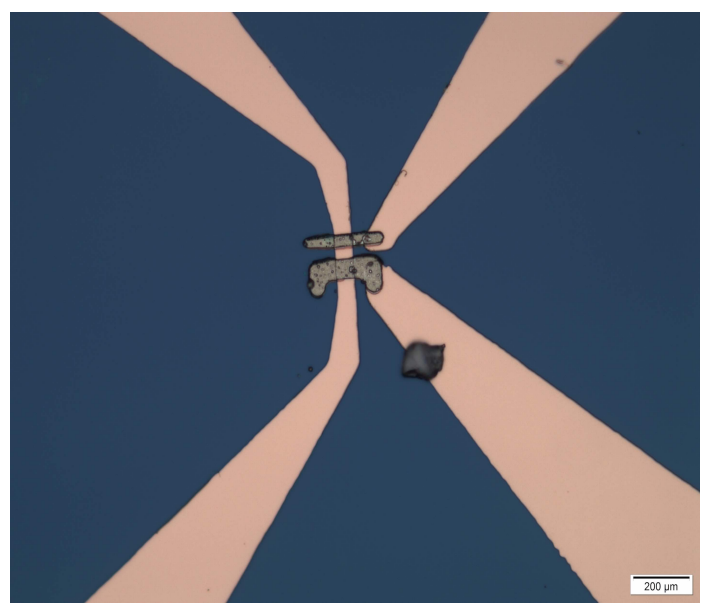

Figure 4.2: Microscope photo of a $5 \mu \mathrm{m}$ device from W3. The capping layer is not transparent and there are droplet-like features on the surface.

The relatively conductively grown $\mathrm{GdN}$ device had a conductive $\mathrm{GaN}$ capping layer. This sample will be referred to as W3 (see figure 4.2). This conductive GaN capping layer had a low resistance of $150 \Omega$. The growth rate of this conductive sample 
was unstable and likely resulted in a metallic-like capping layer, rather than a semiconductor one.

W1 had GdN grown at a nitrogen partial pressure of $2.16 \times 10^{-4}$ mbar, W2 at $2.20 \times$ $10^{-4}$ mbar, and W3 at $5.30 \times 10^{-5}$ mbar. This change in nitrogen pressure that the Gd was exposed to results in a difference in conductivity of the final GdN. As W1 and W2 were grown at a higher nitrogen partial pressure, there are fewer nitrogen vacancies in the final GdN lattice [37]. This results in a lower conductivity of the GdN. W3 was grown at a nitrogen pressure an order of magnitude lower than W1 and W2. A full summary of growth conditions can be seen in table 4.1 below.

Table 4.1: Growth conditions of GdN and capping layers

\begin{tabular}{|c|c|c|c|c|c|}
\hline Sample & $\begin{array}{c}N_{2} \\
\text { Pressure: } \\
\text { GdN }\end{array}$ & $\begin{array}{c}N_{2} \text { Pressure: } \\
\text { Capping } \\
\text { layer }\end{array}$ & $\begin{array}{c}\text { Thickness: } \\
\text { GdN }\end{array}$ & $\begin{array}{c}\text { Thickness: } \\
\text { Capping } \\
\text { layer }\end{array}$ & $\begin{array}{c}\text { Relative } \\
\text { Conductivity }\end{array}$ \\
\hline W1 & $2.16 \times 10^{-4}$ & $2.22 \times 10^{-4}$ & $55 \mathrm{~nm}$ & $30 \mathrm{~nm}$ & Insulating \\
W2 & $2.20 \times 10^{-4}$ & $2.59 \times 10^{-4}$ & $40 \mathrm{~nm}$ & $30 \mathrm{~nm}$ & Insulating \\
W3 & $5.30 \times 10^{-5}$ & $2.28 \times 10^{-4}$ & $40 \mathrm{~nm}$ & $30 \mathrm{~nm}$ & Conductive \\
\hline
\end{tabular}

\subsubsection{Fabrication results for $10 \mu m, 5 \mu m$, and $2 \mu m$ channel lengths}

Consistently $10 \mu \mathrm{m}$ and $5 \mu \mathrm{m}$ devices were able to be fully fabricated on each wafer, although some were unable to be fully realised. Copper deposition and the second layer of photolithographic patterning for the $10 \mu \mathrm{m}$ and $5 \mu \mathrm{m}$ devices were always successful, but lift-off of the GdN layer did not always result in fully separated electrodes (see figure 4.3). For successful spin-injection, the two electrodes need to be seperate and clearly defined.

Fully completed $2 \mu m$ devices were not able to be fabricated using my process. This is likely due to limitations from the wavelength of light used for the lamp in the mask aligner, as $2 \mu m$ is the sterical limit of patterning of the mask aligner used. 


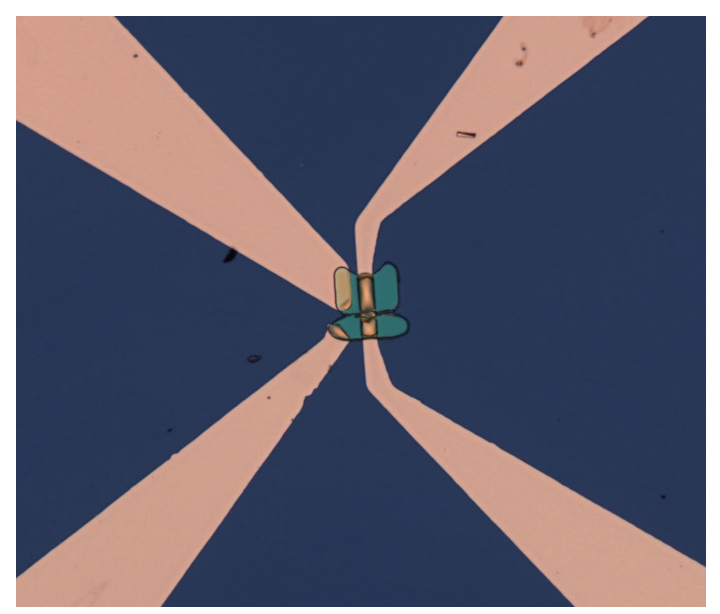

Figure 4.3: Microscope photo of sample with an insulating capping layer of GaN from the same wafer as W2 that didn't successfully complete liftoff after GdN deposition. This device was designed to have a $5 \mu \mathrm{m}$ electrode separation. The electrodes are fused together providing no conducting channel path for spins to diffuse along.

\subsubsection{XRD characterisation for $\mathrm{GdN}$ electrodes}

XRD was used to determine the crystal structure of the films. The squares patterned on the bottom of my wafer only showed peaks from the silicon substrate, as they were too small to be reliably lined up with the x-ray beam. Figure 4.4 shows data from a GdN sample with a GaN capping layer grown on silicon at a nitrogen partial pressure of $3 \times 10^{-4}$ mbar, the same as W2 [58]. The peaks corresponding to GdN are labelled with their Miller indices.

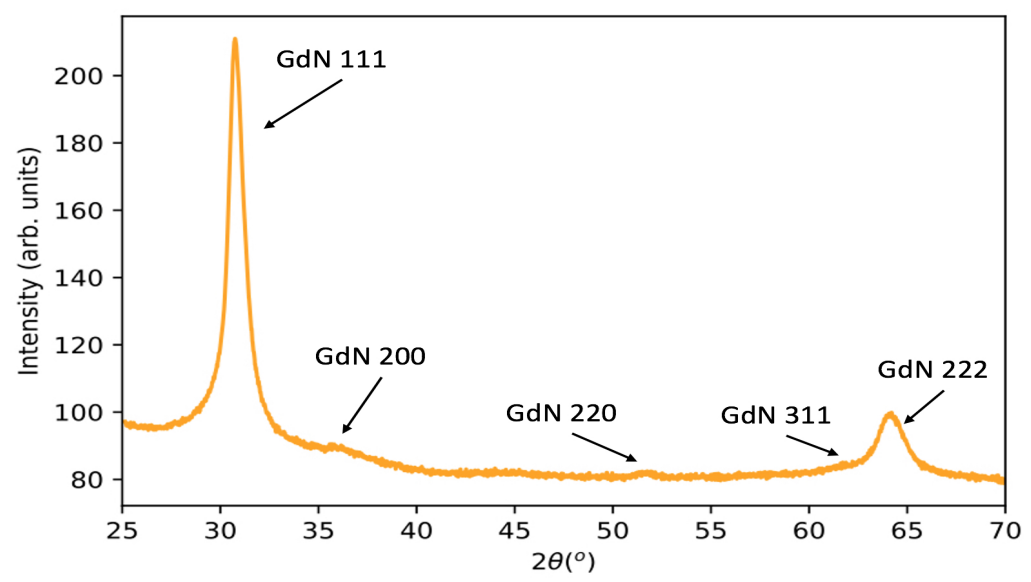

Figure 4.4: XRD data of GdN with GaN capping layer grown in similar conditions to W2. 


\subsubsection{Fabrication results discussion}

The fabrication process encountered several issues within the photolithography process. The developer used became contaminated, as well as the mask aligner lamp needing replacement. Both of these issues presented as a smearing away of the resist pattern towards the centre of my contacts, consequently where my devices were. This resulted in my device pattern not being properly defined. Development of this incomplete pattern also took up to 5 minutes, or approximately 15 times longer than usual (see 3.1). This was solved by both changing the lamp and also by remaking the developer.

Lift-off of the GdN electrodes had to be monitored, as sonication for even 5 minutes proved to damage the electrodes, and rip parts of them off the substrate. Tiny features of the order of my GdN electrodes are more sensitive to the vigorous shaking in sonication, hence the failed lift-off. Samples were instead soaked in acetone for 1-2 hours without sonication, and then checked under the microscope to ensure no residual GdN was left outside the patterned area. If at this point any was apparent, the whole wafer was put in acetone and sonicated for 1 minute before being checked again. This additional caution in lift-off of the GdN electrodes was usually successful in preventing damage to the electrodes.

If the sample did not develop cleanly or took much longer to develop, the developer had likely been contaminated. When this was suspected, developing for 10 seconds at a time and noting the rate of development and whether features can be realised, or testing with another developer (such as AZ Developer MIC) confirmed contamination. In this case, remixing the developer used, and being diligent about the timing of development allowed for realisation of the patterned resist.

\subsubsection{GdN and copper contact results discussion}

The copper contacts appeared to be of good quality as seen under the microscope, and when they were grown they had a consistent deposition rate of $0.4 \AA / s$ as measured by the evaporator's thickness monitor. However, the quality of the GdN was not as certain. The evaporation of the metals for the insulating capping layers was not entirely 
consistent (both the aluminium and galium had varying deposition rates) so sections of the capping layers may be more metallic than others, not creating a uniform electrical environment. The conductively grown GaN capping layer appeared smooth at a macroscopic level, but upon inspection under the microscope, it was apparent that there were droplet like formations on the surface (see figure 4.2). These are likely pure galium or at least gallium rich metal droplets. Their presence suggests a non-uniform deposition, with potential inconsistencies at the GdN-GaN interface.

Another undetermined aspect of fabrication is whether there was a consistent path for electrons between the conducting channel and contacts connected to the GdN electrodes. If the GdN layer was not thick enough, it may not be fully connected down the sides of the copper layer resulting in an inconsistent pathway for the electrons associated with the spin transport.

\subsection{Device testing}

After fabrication was complete, devices were tested to determine the integrity of the electrical contacts both through the spin-injection device and also between the spininjection device and the PPMS rotator puck. Electrical testing was done using handheld ohmmeters, as well as the voltmeters, current source, and breakout box used in the spin injection measurement system setup (see section 3.3 for more details).

Testing done using the handheld meter was to take rough measurements to determine the quality of the copper conducting channel, as well as to determine the order of magnitude of the resistance across all electrical pathways in the spin-injection device; through the copper conducting channel, through one GdN electrode, or through both $\mathrm{GdN}$ electrodes (see figure 4.5). Both GdN electrodes had to be measured to determine their integrity, as both are required for the measurement of the spin current.

Once it is established that the spin-injection device was fully intact and had sufficient electrical contact through all parts of the device, accurate resistance readings of each pathway can be taken using the spin-injection measurement system setup. By controlling the current through the use of the Keithley 6220 Precision Current Source 
(see figure 3.9a), we can also find the IV curve, to determine the dominating electrical behaviour of the pathway through one GdN electrode at room temperature (see figure 4.5).

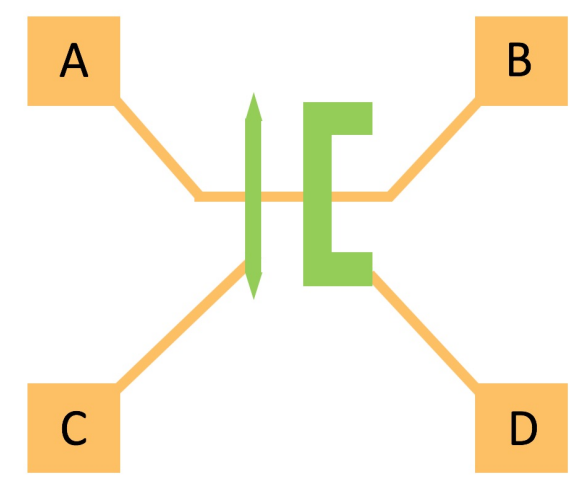

Figure 4.5: Current and voltage measurement geometries were chosen in reference to which contact pads the current would be sent through and where the voltage would be measured. The following electrical tests will be described in reference to A, B, C, and $\mathrm{D}$ as shown here.

\subsubsection{Resistance measurements}

Resistance measurements using the current source and voltmeters were done using both two and three-point resistance measurements. Two-point measurements were done initially using the handheld device to determine the existence of an electrical pathway, whereas three point measurements were done using the spin-injection measurement setup to measure the resistance through each pathway. This was chosen as voltage measured using a three-point geometry has less noise as the resistance of one voltage lead was excluded from the measurement. It was essential to know that the pathways could conduct and had reasonable resistances: if they were too insulating, a reliable current (minimum $\sim 1 \mu A$ ) would not be able to be passed through the device.

Four-point measurements were not done due to the design of my spin-injection device. To measure the electrical voltage, we need the voltage to be measured across two points: one at the same voltage as the start of the electrical current, and the other the end. This cannot be achieved with my spin-injection device geometry. The conducting 
channel connects points $\mathrm{A}$ and $\mathrm{B}$, but points $\mathrm{C}$ and $\mathrm{D}$ are not connected directly; they are connected via two relatively resistive pathways (the $\mathrm{GdN}$ electrodes). If the current goes from $A$ to $C$, then it does not travel through $B$ and $D$. This means that $B$ and $D$ are both at the same voltage, $0 \mathrm{~V}$ relative to each other. Therefore, if a four-point measurement were to be done by sending current from A to $C$, and measuring the voltage across $\mathrm{B}$ and $\mathrm{D}$, the voltage would be measured as $0 \mathrm{~V}$. This is the case with all other four-point configurations. Therefore a three-point IV measurement is the highest accuracy measurement that can be taken.

The resistance measurements of $\mathrm{W} 2$ and $\mathrm{W} 3$ are seen below in tables 4.2 and 4.3 respectively.

\begin{tabular}{|c|c|c|c|}
\hline Part of device & Current Path & Voltage Path & Resistance \\
\hline Second electrode & BC & AD & $25 k \Omega$ \\
Second electrode & AD & BD & $27 k \Omega$ \\
Conducting channel & AD & AB & $32.5 \Omega$ \\
First electrode & AD & AC & Off scale \\
\hline
\end{tabular}

Table 4.2: Resistance measurements for W2. Refer to figure 4.5 for geometries.

\begin{tabular}{|c|c|c|c|}
\hline Part of device & Current Path & Voltage Path & Resistance \\
\hline First electrode & AD & AC & $175 \Omega$ \\
Second electrode & BC & BD & $62 \Omega$ \\
Conducting channel & AD & AB & $34 \Omega$ \\
\hline
\end{tabular}

Table 4.3: Resistance measurements for W3. Refer to figure 4.5 for geometries.

Across all samples, the copper conducting channel and contact pads had a resistance of $33 \pm 4 \Omega$. This indicates lithography was successful at fabricating the copper conducting channel and contact pads. When there was an unreliable electrical path through the GdN electrodes, a resistance of over $1 M \Omega$ was measured. In these cases, no currents higher than $1 n A$ could be sent through the spin-injection device. These off-scale resistance values were likely caused by oxidation of the $\mathrm{GdN}$ or a disconnect between the GdN electrode and the copper conducting channel or copper contacts, all resulting in a highly insulating electrical pathway. W2 had more resistive pathways 
through the GdN electrodes than W3. This is due to W2 being grown to be less conductive than W3, and also having an insulating capping layer. W3 was grown to be more conductive so has a less resistive GdN. However, as the resistances measured in W3 are what is expected of a metallic-like material, the current likely travelled through the capping layer as well, not just the GdN. W3 was the only device that had all electrical pathways intact enough to give a resistance measurement across all of them. W1 and W2 had one GdN pathway that had off-scale resistance.

\section{Temperature dependent resistance measurement}

Using the PPMS, the resistance as a function of temperature was measured for $\mathrm{W} 3$ and is summarised in table 4.4 below. The purpose of this was to determine whether the pathway through the electrodes was predominantly semiconducting or more metallic.

\begin{tabular}{|c|c|}
\hline Temperature & Resistance \\
\hline $300 \mathrm{~K}$ & $12.72 \Omega$ \\
$107.5 \mathrm{~K}$ & $10.58 \Omega$ \\
\hline
\end{tabular}

Table 4.4: Resistance vs. temperature measurements for W3.

High accuracy was not required for this measurement as it was to gauge the general behaviour of the device when exposed to different temperatures. The current source of $1 m A$ travelled from $C$ to $D$ (see figure 4.5), and the voltage was measured across $C$ and $\mathrm{D}$ making this a two-point measurement. The resistance is seen to decrease with temperature indicating that the behaviour of the pathway through both GdN electrodes is predominantly metallic. This is likely due to the high conductivity of the GaN capping layer and copper contacts and conducting channel dominating the measurable resistance 


\subsubsection{IV measurements}

Initial IV measurements were taken across both of the ferromagnetic electrodes in W3 as seen in figure 4.6 .

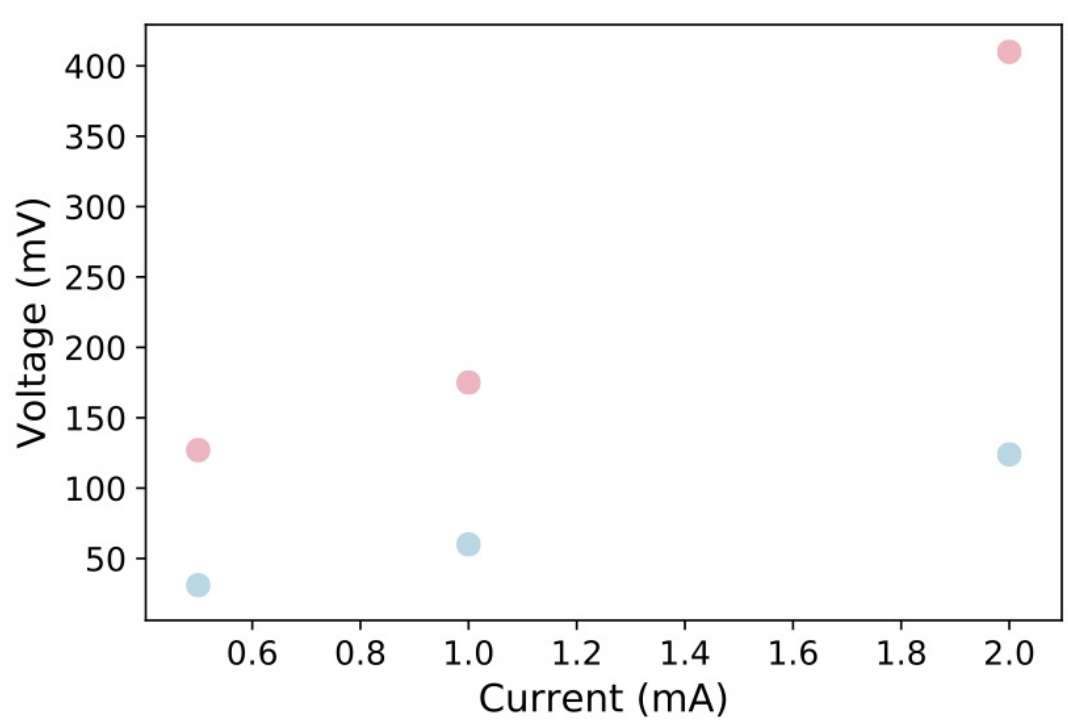

Figure 4.6: IV data from W3. Pink is data from across the first electrode, blue is from the second. See table 4.3 for details on the geometry used.

The second electrode shows a more linear relationship between the current and voltage, suggesting it is more metallic-like. The first electrode has an increase in voltage at $2 \mu \mathrm{A}$, suggesting a more semi-conducting pathway across that junction.

\subsubsection{Discussion of electrical testing results}

Interestingly, W3 having a metallic-like GaN capping layer had all electrical contacts functioning, whereas the other samples capped with insulating GaN did not have readable voltages through one of the GdN electrodes. It could be argued that this is because of the metallic-like electrical properties of the relatively conducting GaN providing an easier and more intact path for the electrical current to travel through than just the comparatively insulating $\mathrm{GdN}$, the context of these films growths need to be taken into account. 
W3 was grown the day before testing and had minimal atmosphere exposure during that time. This device was fabricated under perfect conditions and had clean edges on all features. The GdN was purposely grown to be more conductive. W1 and W2 were fabricated up to a week before being tested, and W2 was exposed to the atmosphere for nearly 12 hours. Even though the GdN electrodes didn't oxidise entirely, edges of the GdN that weren't fully encased by the capping layer may have begun to, inhibiting a full range of pathways for the current. The contact between the electrodes and the copper was only $5 \mu \mathrm{m}$ wide in places, so even minimal oxidation could have a substantial impact.

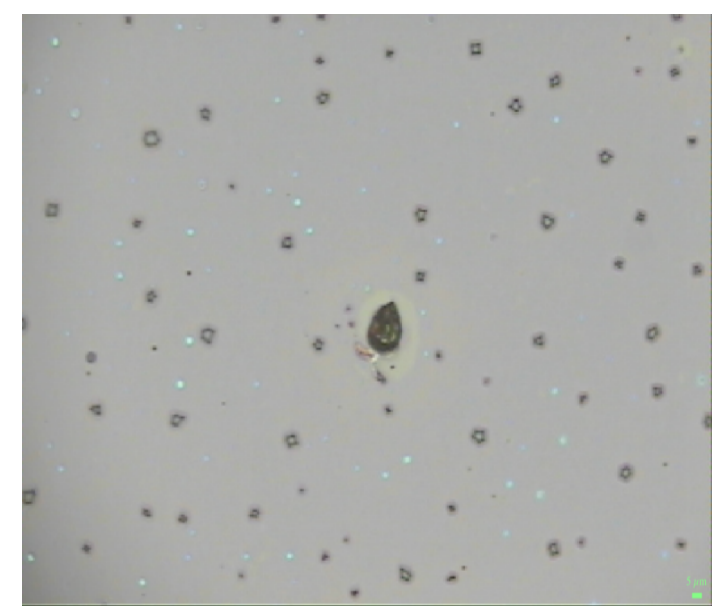

Figure 4.7: Oxidation on the surface of a samarium nitride sample [36].

W2 under a microscope (see figure 4.1) had no observable oxidation on the surface. Oxidation appears as flaky metallic spots across the surface of the GdN (see figure 4.7). An alternative is that the GdN layer was not fully connected across entire growth. The deposition is designed to be uniform and perpendicular to the substrate. However, if the GdN layer is not as thick as the chrome and copper, it may not connect across the whole electrode (see figure 4.8). Although the GdN may be non-continuous, there is still a path for the electrons through the conducting copper. However, the separated GdN could be more susceptible to oxidation at points where the copper and GdN need to come into contact, degrading the spin-injection device. Due to the different shapes of the electrodes, it is possible that this may only occur in one out of the two electrodes, and could explain the inconsistency seen in some devices. 


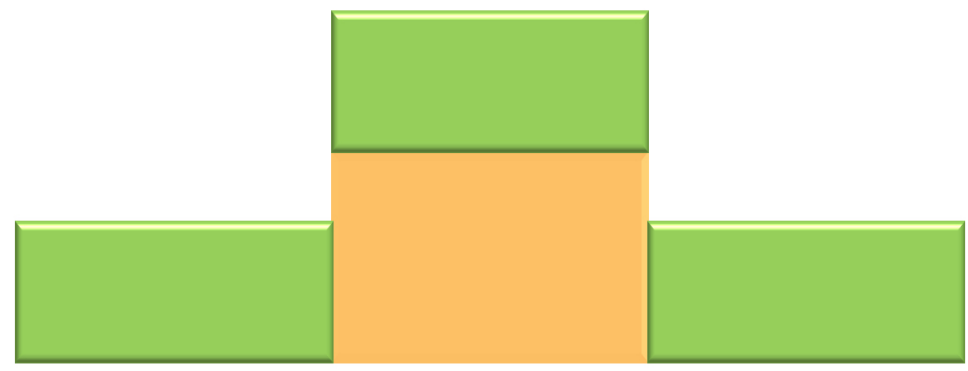

Figure 4.8: The green GdN layer is not as thick as the orange copper, so the GdN electrode is not continuous across the copper conducting channel/contact pads.

For preliminary testing, a highly conductive GdN layer with an insulating capping layer would be the best choice. If a conductive or metallic-like capping layer is used, it can be difficult to distinguish the resistivity and functionality of the GdN, as the overall resistance of the system is determined by that of the capping layer. This may also be the case for future spin-injection measurements, as if the electrons are predominantly transported through the metallic-like capping layer, it may present issues regarding how much of the injected current is spin-polarised. However, this will require more investigation before a conclusion can be reached.

\subsubsection{Durability of samples in testing}

There was a tendency for the samples to increase resistance by three orders of magnitude within a few days. IV measurements were taken of W3 once this was seen to occur. This was done using the voltmeters and current source from the spin injection measurement set-up for $\mathrm{W} 3$ across the second GdN electrode (see figure 4.9). These were taken after W3 was removed from the PPMS.

Current went from A to D, with the voltage being measured across B and D (see figure 4.5). As seen previously in figure 4.6 as the pink data set, this testing configuration initially suggested a non-linear relationship between the current and voltage. However in figure 4.9, the data is linear, indicating metallic-like behaviour of this pathway. A maximum of $10.3 \mu A$ was able to be sent through this pathway, compared to the several $m A$ in figure 4.6. W3 was then looked at under the microscope after this IV measurement was taken. 


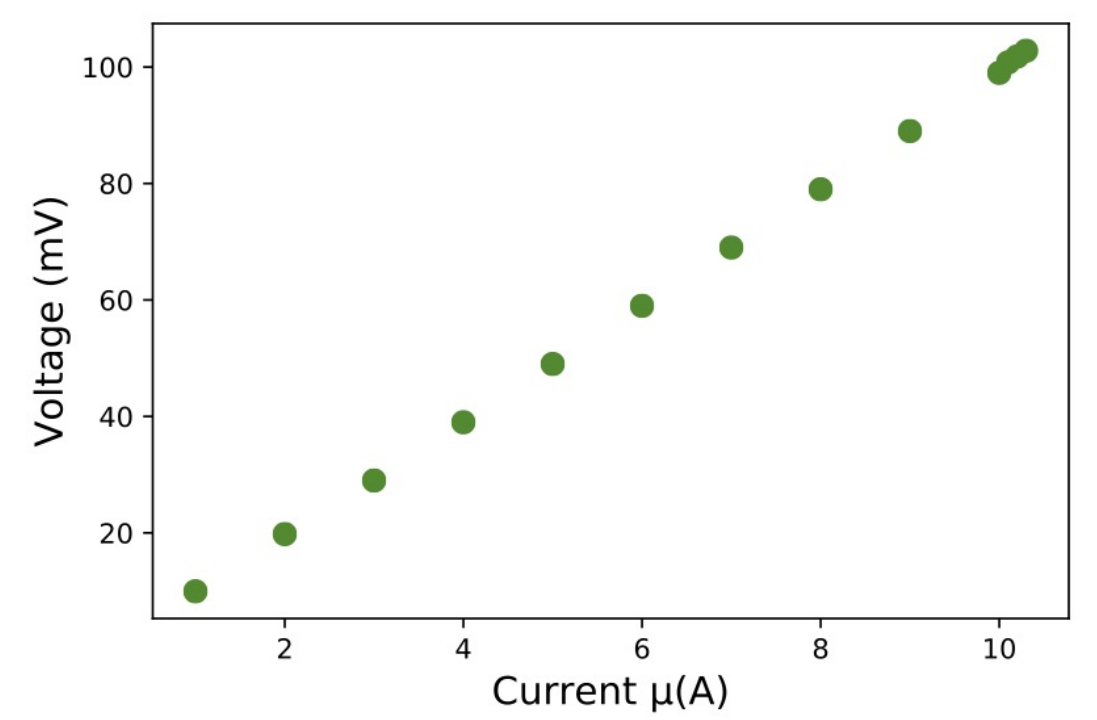

Figure 4.9: Data collected from W3 after being in the PPMS.

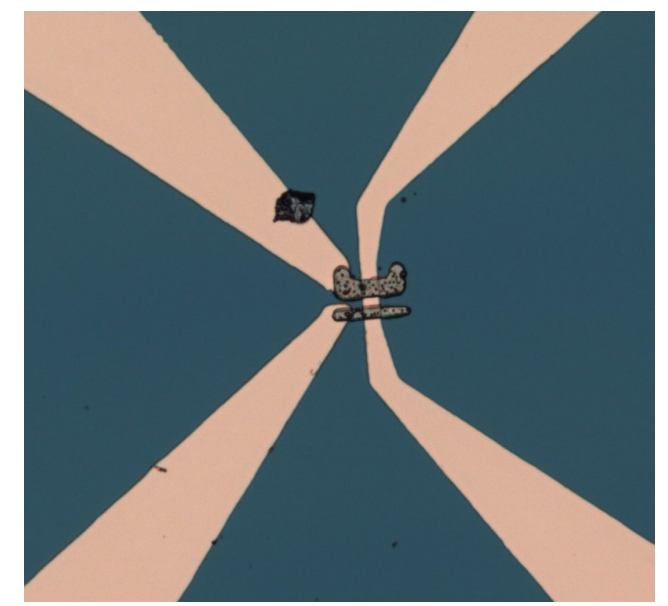

(a) W3 before PPMS

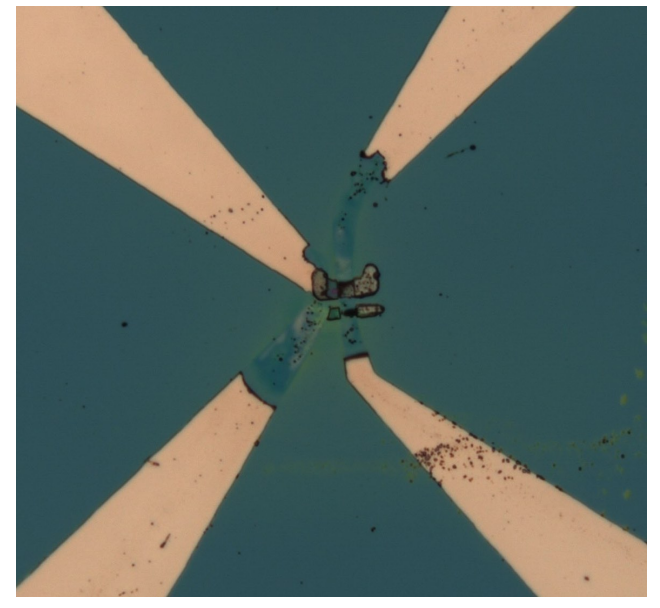

(b) W3 after PPMS

Figure 4.10: Difference in spin injection device W3 before and after being measured in the PPMS. Three days separate these two microscope pictures.

Upon inspection under the microscope, the GdN electrodes are mostly intact, and it is predominantly the copper that came off (see figure $4.10 \mathrm{~b}$ ). This shows that the GdN electrodes adhere well to the $\mathrm{Si} / \mathrm{SiO}_{2}$ substrate, more so than the chrome and copper. There are several possibilities into what caused the chrome and copper to be removed 
from the substrate. One is that it is the result of the temperature-dependent measurements reported in section 4.2.1 that were done in the PPMS. There is still some residue of the conducting channel and connections to the contact pads (see figure $4.10 \mathrm{~b}$ ), so there may still be chrome on the substrate and it may be that just the thicker copper layer peeled off. This may be due to a mismatch between the thermal coefficient of expansion for the $\mathrm{Si} / \mathrm{SiO}_{2}$ substrate and the copper. The thermal coefficient of copper is $16.5 \mu \mathrm{mm}^{-1} \mathrm{~K}^{-1}$ [59] which is eight times higher than that of silicon, $2.6 \mu \mathrm{mm}^{-1} \mathrm{~K}^{-1}$ [59]. This means that the copper could have shrunk up to eight times as much as the silicon when being cooled and had to expand the same amount when heated. The temperature change during the RT measurement was relatively fast at $10 \mathrm{~K} / \mathrm{min}$, so a build-up of stress from thermal expansion is likely. In the future, changing the temperature more slowly could avoid the build-up of stress that may have caused the copper to peel off. 


\section{Chapter 5}

\section{Conclusions and Future Work}

A spin-injection device using $\mathrm{GdN}$ as the material for the ferromagnetic electrodes was designed and fabricated using photolithography, thermal evaporation, and physical vapour deposition. Fabrication issues were identified and an optimal method was determined. These devices were characterised and then electrically tested to determine their resistance and electrical integrity. It was found that the copper conducting channel had a consistent resistance of $33 \pm 4 \Omega$, and that devices with a conducting channel length of $5 \mu \mathrm{m}$ and $10 \mu \mathrm{m}$ could be successfully fabricated, whilst those with $2 \mu m$ conducting channel lengths could not. Devices often had only one GdN electrode intact enough to provide a usable electrical pathway. This is possibly due to oxidation, highly insulating GdN electrodes, or insufficient deposition to create fully connected GdN electrodes.

To further this project towards a fully realisable spin-injection transistor, developments to my design need to be made. These could include ensuring durability of copper in temperature dependent experiments, developing the design of the spin-injection device to allow for smaller features to be patterned, and moving on to use electronbeam lithography for REN patterning. An investigation into the applicability of a range of GdN conductivities would be useful, as well as determining the overall influence of the capping layer on the electrical pathways, and how this might affect the polarisation of the injected current. 


\section{Bibliography}

[1] R. Danilak, "Why Energy Is A Big And Rapidly Growing Problem For Data Centers," 2017. [Online]. Available: $\quad$ https://www.forbes.com/sites/forbestechcouncil/2017/12/15/ why-energy-is-a-big-and-rapidly-growing-problem-for-data-centers $/\{\#\}$ $11366 f 7 d 5 a 30$

[2] A. Vaughan, "How viral cat videos are warming the planet," 2015. [Online]. Available: https://www.theguardian.com/environment/2015/sep/25/ server-data-centre-emissions-air-travel-web-google-facebook-greenhouse-gas

[3] P. Greaves, "Data centres - the world's greatest energy guzzlers." [Online]. Available: https://www. aurecongroup.com/thinking/insights/data-centres-for-the-digital-age/ data-centres-the-worlds-greatest-energy-guzzlers

[4] R. K. Cavin, P. Lugli, and V. V. Zhirnov, "Science and engineering beyond Moore's law," Proceedings of the IEEE, vol. 100, no. Special Centennial Issue, pp. 1720-1749, 2012.

[5] S. Datta and B. Das, "Electronic analog of the electro-optic modulator," Applied Physics Letters, vol. 56, no. 7, pp. 665-667, 1990. [Online]. Available: https://doi.org/10.1063/1.102730

[6] S. Sato, M. Tanaka, and R. Nakane, "Spin transport in Si-based spin metaloxide-semiconductor field-effect transistors: Spin drift effect in the inversion channel and spin relaxation in the $\mathrm{n}+-\mathrm{Si}$ source/drain regions," arXiv preprint arXiv:2003.02415, 2020.

[7] B. M. Ludbrook, I. L. Farrell, M. Kuebel, B. J. Ruck, A. R. H. Preston, H. J. Trodahl, L. Ranno, R. J. Reeves, and S. M. Durbin, "Growth and properties of 
epitaxial GdN," Journal of Applied Physics, vol. 106, no. 6, p. 63910, 2009. [Online]. Available: https://doi.org/10.1063/1.3211290

[8] F. J. Jedema, H. B. Heersche, A. T. Filip, J. J. A. Baselmans, and B. J. van Wees, "Electrical detection of spin precession in a metallic mesoscopic spin valve," Nature, vol. 416, no. 6882, pp. 713-716, 2002. [Online]. Available: https:/ / doi.org/10.1038/416713a

[9] I. Žutić, J. Fabian, and S. Das Sarma, "Spintronics: Fundamentals and applications," Rev. Mod. Phys., vol. 76, no. 2, pp. 323-410, apr 2004. [Online]. Available: https://link.aps.org/doi/10.1103/RevModPhys.76.323

[10] V. Medeliene and V. Stankevič, "The influence of particles of carbon nanoderivative on the properties of the electrocodeposited copper nanocomposite coating," Galvanotechnik, vol. 103, pp. 942-946, 2012.

[11] F. Natali, B. J. Ruck, N. O. V. Plank, H. J. Trodahl, S. Granville, C. Meyer, and W. R. L. Lambrecht, "Rare-earth mononitrides," Progress in Materials Science, vol. 58, no. 8, pp. 1316-1360, 2013. [Online]. Available: http: //www.sciencedirect.com/science/article/pii/S007964251300056X

[12] D. D. Awschalom and J. M. Kikkawa, "Electron Spin and Optical Coherence in Semiconductors," Physics Today, vol. 52, no. 6, pp. 33-38, jun 1999. [Online]. Available: http://physicstoday.scitation.org/doi/10.1063/1.882695

[13] S. A. Wolf, D. D. Awschalom, R. A. Buhrman, J. M. Daughton, v. S. von Molnár, M. L. Roukes, A. Y. Chtchelkanova, and D. M. Treger, "Spintronics: a spin-based electronics vision for the future," Science, vol. 294, no. 5546, pp. 1488-1495, 2001.

[14] G. Binasch, P. Grünberg, F. Saurenbach, and W. Zinn, "Enhanced magnetoresistance in layered magnetic structures with antiferromagnetic interlayer exchange," Phys. Rev. B, vol. 39, no. 7, pp. 4828-4830, mar 1989. [Online]. Available: https://link.aps.org/doi/10.1103/PhysRevB.39.4828

[15] M. N. Baibich, J. M. Broto, A. Fert, F. N. Van Dau, F. Petroff, P. Etienne, G. Creuzet, A. Friederich, and J. Chazelas, "Giant Magnetoresistance of (001)Fe/(001)Cr Magnetic Superlattices," Phys. Rev. Lett., vol. 61, no. 21, pp. 2472-2475, nov 1988. [Online]. Available: https://link.aps.org/doi/10.1103/PhysRevLett.61.2472 
[16] S. M. Sze, Semiconductor Devices: Physics and Technology, 2nd ed. Wiley, 2002.

[17] S. Bhatti, R. Sbiaa, A. Hirohata, H. Ohno, S. Fukami, and S. N. Piramanayagam, "Spintronics based random access memory: a review," Materials Today, vol. 20, no. 9, pp. 530-548, 2017. [Online]. Available: http://www.sciencedirect.com/ science/article/pii/S1369702117304285

[18] J. S. Friedman, B. W. Wessels, G. Memik, and A. V. Sahakian, "Emitter-Coupled Spin-Transistor Logic: Cascaded Spintronic Computing Beyond $10 \mathrm{GHz}$," IEEE Journal on Emerging and Selected Topics in Circuits and Systems, vol. 5, no. 1, pp. 17-27, 2015.

[19] Traditional Planar MOSFETs. John Wiley \& Sons, Ltd, 2016, ch. 3, pp. 231-294. [Online]. Available: https://onlinelibrary.wiley.com/doi/abs/10.1002/ 9781119155850.ch3

[20] From Scaling-Driven Technological Variations to Novel Dimensions in MISFETs. John Wiley \& Sons, Ltd, 2016, ch. 4, pp. 295-374. [Online]. Available: https: //onlinelibrary.wiley.com/doi/abs/10.1002/9781119155850.ch4

[21] Rogerb, "FET cross section," 2005. [Online]. Available: https://commons. wikimedia.org/wiki/File:FET\{_\}cross\{_\}section.png

[22] BYJU's The Learning App, "Transistor: NPN Transistor And PNP Transistor." [Online]. Available: https://byjus.com/physics/actions-of-transistor/

[23] D. Laws, "Who Invented The Transistor?" 2013. [Online]. Available: https://computerhistory.org/blog/who-invented-the-transistor/?key= who-invented-the-transistor

[24] S. Datta, "How we proposed the spin transistor," Nature Electronics, vol. 1, no. 11, p. 604, 2018. [Online]. Available: https://doi.org/10.1038/s41928-018-0163-4

[25] S. Maekawa, Ed., Concepts in Spin Electronics. New York: Oxford University Press, 2006.

[26] H. C. Koo, J. H. Kwon, J. Eom, J. Chang, S. H. Han, and M. Johnson, "Control of Spin Precession in a Spin-Injected Field Effect Transistor," Science, vol. 325, no. 5947, pp. 1515-1518, 2009. [Online]. Available: https: //science.sciencemag.org/content/325/5947/1515 
[27] K. C. Hall and M. E. Flatté, "Performance of a spin-based insulated gate field effect transistor," Applied Physics Letters, vol. 88, no. 16, p. 162503, 2006. [Online]. Available: https://doi.org/10.1063/1.2192152

[28] G. Schmidt, D. Ferrand, L. W. Molenkamp, A. T. Filip, and B. J. van Wees, "Fundamental obstacle for electrical spin injection from a ferromagnetic metal into a diffusive semiconductor," Phys. Rev. B, vol. 62, no. 8, pp. R4790-R4793, aug 2000. [Online]. Available: https://link.aps.org/doi/10.1103/PhysRevB.62. R4790

[29] Y. K. Ko, D. S. Park, B. S. Seo, H. J. Yang, H. J. Shin, J. Y. Kim, J. H. Lee, W. H. Lee, P. J. Reucroft, and J. G. Lee, "Studies of cobalt thin films deposited by sputtering and MOCVD," Materials Chemistry and Physics, vol. 80, no. 2, pp. 560-564, 2003. [Online]. Available: http: / / www.sciencedirect.com/science/article/pii/S0254058403000853

[30] MTI, “MTI Cooperation," 2020. [Online]. Available: https://www.mtixtl.com/ ganepitaxialtemplateonsapphirecplanentypeundoped2x5micron-4-1-1-1.aspx

[31] R. Jansen, S. P. Dash, S. Sharma, and B. C. Min, "Silicon spintronics with ferromagnetic tunnel devices," Semiconductor Science and Technology, vol. 27, no. 8, p. 83001, jul 2012. [Online]. Available: https://doi.org/10.1088\{\%\} 2F0268-1242\{\%\}2F27\{\%\}2F8\{\%\}2F083001

[32] N. Matsuo, N. Doko, T. Takada, H. Saito, and S. Yuasa, "High Magnetoresistance in Fully Epitaxial Magnetic Tunnel Junctions with a Semiconducting GaOx Tunnel Barrier," Phys. Rev. Applied, vol. 6, no. 3, p. 34011, sep 2016. [Online]. Available: https://link.aps.org/doi/10.1103/PhysRevApplied.6.034011

[33] A. Spiesser, H. Saito, S. Yuasa, and R. Jansen, "Tunnel spin polarization of $\mathrm{Fe} / \mathrm{MgO} / \mathrm{Si}$ contacts reaching 90\% with increasing $\mathrm{MgO}$ thickness," Phys. Rev. B, vol. 99, no. 22, p. 224427, jun 2019. [Online]. Available: https:/ /link.aps.org/doi/10.1103/PhysRevB.99.224427

[34] T. Kanaki, H. Asahara, S. Ohya, and M. Tanaka, "Spin-dependent transport properties of a GaMnAs-based vertical spin metal-oxide-semiconductor fieldeffect transistor structure," Applied Physics Letters, vol. 107, no. 24, p. 242401, 2015. [Online]. Available: https: / / doi.org/10.1063/1.4937437 
[35] C.-G. Duan, R. F. Sabirianov, W. N. Mei, P. A. Dowben, S. S. Jaswal, and E. Y. Tsymbal, "Electronic, magnetic and transport properties of rare-earth monopnictides," Journal of Physics: Condensed Matter, vol. 19, no. 31, p. 315220, jul 2007. [Online]. Available: https://doi.org/10.1088\{\%\}2F0953-8984\{\%\}2F19\{\%\} 2 F31 $\%$ \% 2 F315220

[36] K. Pitman, "Raman Spectroscopy of Rare Earth Nitrides," Honours, Victoria University of Wellington, 2018.

[37] C.-M. Lee, H. Warring, S. Vézian, B. Damilano, S. Granville, M. Al Khalfioui, Y. Cordier, H. J. Trodahl, B. J. Ruck, and F. Natali, "Highly resistive epitaxial Mg-doped GdN thin films," Applied Physics Letters, vol. 106, no. 2, p. 22401, 2015. [Online]. Available: https://doi.org/10.1063/1.4905598

[38] N. O. V. Plank, F. Natali, J. Galipaud, J. H. Richter, M. Simpson, H. J. Trodahl, and B. J. Ruck, "Enhanced Curie temperature in N-deficient GdN," Applied Physics Letters, vol. 98, no. 11, p. 112503, 2011. [Online]. Available: https:/ / doi.org/10.1063/1.3566996

[39] A. Pal, K. Senapati, Z. H. Barber, and M. G. Blamire, "Electric-FieldDependent Spin Polarization in GdN Spin Filter Tunnel Junctions," Advanced Materials, vol. 25, no. 39, pp. 5581-5585, 2013. [Online]. Available: https: //onlinelibrary.wiley.com/doi/abs/10.1002/adma.201300636

[40] F. Natali, B. Ludbrook, J. Galipaud, N. Plank, S. Granville, A. Preston, B. L. Do, J. Richter, I. Farrell, R. Reeves, and Others, "Epitaxial growth and properties of GdN, EuN and SmN thin films," physica status solidi c, vol. 9, no. 3-4, pp. 605-608, 2012.

[41] M. A. Scarpulla, C. S. Gallinat, S. Mack, J. S. Speck, and A. C. Gossard, “GdN $\left(\begin{array}{lll}1 & 1 & 1\end{array}\right)$ heteroepitaxy on GaN (lo $\left.00 \begin{array}{lll}0 & 0\end{array}\right)$ by N2 plasma and NH3 molecular beam epitaxy," Journal of Crystal Growth, vol. 311, no. 5, pp. 1239-1244, 2009.

[42] H. J. Trodahl, A. R. H. Preston, J. Zhong, B. J. Ruck, N. M. Strickland, C. Mitra, and W. R. L. Lambrecht, “Ferromagnetic redshift of the optical gap in GdN," Physical Review B, vol. 76, no. 8, p. 85211, 2007.

[43] R. Ohshima, S. Klingler, S. Dushenko, Y. Ando, M. Weiler, H. Huebl, T. Shinjo, S. T. B. Goennenwein, and M. Shiraishi, "Spin injection into 
silicon detected by broadband ferromagnetic resonance spectroscopy," Applied Physics Letters, vol. 110, no. 18, p. 182402, 2017. [Online]. Available: https: / / doi.org/10.1063/1.4983012

[44] H. Ding, Y. Gao, M. Cinchetti, J.-P. Wüstenberg, M. Sánchez-Albaneda, O. Andreyev, M. Bauer, and M. Aeschlimann, "Spin injection and spin dynamics at the $\mathrm{CuPc} / \mathrm{GaAs}$ interface studied with ultraviolet photoemission spectroscopy and two-photon photoemission spectroscopy," Phys. Rev. B, vol. 78, no. 7, p. 75311, aug 2008. [Online]. Available: https://link.aps.org/doi/10.1103/ PhysRevB.78.075311

[45] S. Ringer, M. Rosenauer, T. Völkl, M. Kadur, F. Hopperdietzel, D. Weiss, and J. Eroms, "Spin field-effect transistor action via tunable polarization of the spin injection in a Co/MgO/graphene contact," Applied Physics Letters, vol. 113, no. 13, p. 132403, 2018. [Online]. Available: https://doi.org/10.1063/1.5049664

[46] S. Omar and B. J. van Wees, "Graphene-WS2 heterostructures for tunable spin injection and spin transport," Phys. Rev. B, vol. 95, no. 8, p. 81404, feb 2017. [Online]. Available: https://link.aps.org/doi/10.1103/PhysRevB.95.081404

[47] M. Buchner, T. Kuczmik, M. Oltscher, M. Ciorga, T. Korn, J. Loher, D. Schuh, C. Schüller, D. Bougeard, D. Weiss, and C. H. Back, "Optical investigation of electrical spin injection into an inverted two-dimensional electron gas structure," Phys. Rev. B, vol. 95, no. 3, p. 35304, jan 2017. [Online]. Available: https:/ /link.aps.org/doi/10.1103/PhysRevB.95.035304

[48] A. Hirohata, Y. Baba, B. A. Murphy, B. Ng, Y. Yao, K. Nagao, and J.-y. Kim, "Magneto-optical detection of spin accumulation under the influence of mechanical rotation," Scientific Reports, vol. 8, no. 1, p. 1974, 2018. [Online]. Available: https://doi.org/10.1038/s41598-018-20269-5

[49] H. Idzuchi, Y. Fukuma, and Y. Otani, "Spin transport in non-magnetic nano-structures induced by non-local spin injection," Physica E: Low-dimensional Systems and Nanostructures, vol. 68, pp. 239-263, 2015. [Online]. Available: http:/ / www.sciencedirect.com/science/article/pii/S1386947714004305

[50] S. Takahashi and S. Maekawa, "Spin injection and detection in magnetic nanostructures," Physical Review B, vol. 67, no. 5, p. 52409, 2003. 
[51] Y. Otani and T. Kimura, "Manipulation of spin currents in metallic systems," Philosophical Transactions of the Royal Society A: Mathematical, Physical and Engineering Sciences, vol. 369, no. 1948, pp. 3136-3149, 2011. [Online]. Available: https://royalsocietypublishing.org/doi/abs/10.1098/rsta.2011.0010

[52] Layout Editor, “Layout Editor.” [Online]. Available: https://layouteditor.com/

[53] L. A. Browning, "Spatial network conduction in carbon nanotube and Ag Ag2S Ag atomic switch network device platforms," 2019. [Online]. Available: https://drive.google.com/drive/u/0/folders/1he14X-NVq5\{_\} yq6Ai0RCkz13JXtpo7SD1

[54] S. Granville, B. J. Ruck, F. Budde, A. Koo, D. J. Pringle, F. Kuchler, A. R. H. Preston, D. H. Housden, N. Lund, A. Bittar, G. V. M. Williams, and H. J. Trodahl, "Semiconducting ground state of GdN thin films," Phys. Rev. B, vol. 73, no. 23, p. 235335, jun 2006. [Online]. Available: https://link.aps.org/doi/10.1103/PhysRevB.73.235335

[55] C. Kittel, Introduction To Solid State Physics, 8th ed., S. Johnson, Ed. John Wiley \& Sons, Ltd, 2005.

[56] Czech and Slovak Crystallographic Association, "User D8 Advance." [Online]. Available: https://www.xray.cz/kfkl-osa/eng/d8/default.htm

[57] National Instruments, "LabVIEW." [Online]. Available: https://www.ni.com/ en-nz/shop/labview.html

[58] A. Shaib, F. Natali, J. R. Chan, F. Ullstad, W. F. Holmes-Hewett, J. D. Miller, B. J. Ruck, and H. J. Trodahl, "Coexisting structural phases in the catalytically driven growth of rock salt $\{\mathrm{GdN}\}$," Materials Research Express, vol. 7, no. 4, p. 46404, apr 2020. [Online]. Available: https://doi.org/10.1088\{\%\}2F2053-1591\{\%\}2Fab802f

[59] "CRC Handbook of Chemistry and Physics, 84th Edition," D. R. Lide, Ed. Florida: CRC Press, 2003, ch. 12. 